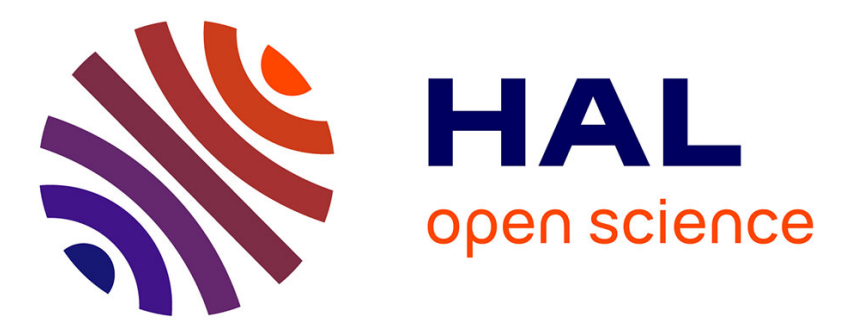

\title{
Histoire Épistémologie Langage De la grammaire générative à la linguistique cognitive : retour sur un basculement théorique
}

Jean-Michel Fortis

\section{- To cite this version:}

Jean-Michel Fortis. Histoire Épistémologie Langage De la grammaire générative à la linguistique cognitive: retour sur un basculement théorique. Histoire Epistémologie Langage, 2012, XXXIV (1), pp.115 - 154. 10.3406/hel.2012.3239 . hal-01417553

\section{HAL Id: hal-01417553 \\ https://hal.science/hal-01417553}

Submitted on 15 Dec 2016

HAL is a multi-disciplinary open access archive for the deposit and dissemination of scientific research documents, whether they are published or not. The documents may come from teaching and research institutions in France or abroad, or from public or private research centers.
L'archive ouverte pluridisciplinaire HAL, est destinée au dépôt et à la diffusion de documents scientifiques de niveau recherche, publiés ou non, émanant des établissements d'enseignement et de recherche français ou étrangers, des laboratoires publics ou privés. 
De la grammaire générative à la linguistique cognitive : retour sur un basculement théorique

Jean-Michel Fortis

\section{Citer ce document / Cite this document :}

Fortis Jean-Michel. De la grammaire générative à la linguistique cognitive : retour sur un basculement théorique. In: Histoire Épistémologie Langage, tome 34, fascicule 1, 2012. La linguistique cognitive : histoire et épistémologie. pp. 115154;

doi : $10.3406 /$ hel.2012.3239

http://www.persee.fr/doc/hel_0750-8069_2012_num_34_1_3239

Document généré le 15/06/2016 


\begin{abstract}
This paper describes the birth of this theoretical conglomerate known as cognitive linguistics. In today's parlance, " cognitive linguistics" refers to a movement that was born in the United Sates in the 1970s and progressively took shape in the work of linguists who wished to break away from syntaxcentered tranformational grammar. This split was to a large extent prepared by the movement of generative semantics, which had steered a number of linguists in a more semantically oriented direction. Generative semantics is given its due in the first part of the paper, in which I analyze the motivations of this movement, and the ways in which it may have prepared the ground for cognitive linguistics. The second part recounts how topics and positions characteristic of cognitive linguistics have emerged and gained momentum. Four central themes are given special emphasis : the notion of conceptual metaphor, the revival of localist ideas, questions related to prototype theory and categorization, and, finally, the concept of frame. In conclusion, it is pointed out that cognitive linguistics gained its unity by weaving together themes and theoretical views of various origins around an empiricist core.
\end{abstract}

\title{
Résumé
}

Cet article décrit la naissance de ce conglomérat théorique que constitue la linguistique cognitive. Les termes de linguistique cognitive renvoient aujourd'hui à un mouvement né aux États-Unis dans les années 1970, et qui a pris forme dans les travaux de linguistes qui souhaitaient rompre avec la grammaire transformationnelle syntactocentrée. Cette rupture a été dans une large mesure préparée au sein de la sémantique générative, qui avait déjà réorienté certains linguistes vers des considérations sémantiques. Les raisons de l'avènement de la sémantique générative et sa contribution à la linguistique cognitive sont analysées dans la première partie. La seconde partie décrit l'émergence des thématiques et des positions caractéristiques de la linguistique cognitive. Quatre thématiques font l'objet d'un examen particulier : la notion de métaphore conceptuelle, la résurgence d'idées localistes, les questions liées à la théorie du prototype et à la catégorisation, et, pour finir, le concept de cadre (frame). En conclusion, on souligne que la linguiste cognitive doit son unité au fait d'avoir construit autour d'un noyau central empiriste un réseau de thématiques et de positions théoriques interconnectées. 


\title{
DE LA GRAMMAIRE GÉNÉRATIVE À LA LINGUISTIQUE COGNITIVE : RETOUR SUR UN BASCULEMENT THÉORIQUE
}

\author{
Jean-Michel Fortis
}

CNRS UMR7597 - PRES Sorbonne Paris Cité

\section{Résumé}

Cet article décrit la naissance de ce conglomérat théorique que constitue la linguistique cognitive. Les termes de linguistique cognitive renvoient aujourd'hui à un mouvement né aux États-Unis dans les années 1970, et qui a pris forme dans les travaux de linguistes qui souhaitaient rompre avec la grammaire transformationnelle syntactocentrée. Cette rupture a été dans une large mesure préparée au sein de la sémantique générative, qui avait déjà réorienté certains linguistes vers des considérations sémantiques. Les raisons de l'avènement de la sémantique générative et sa contribution à la linguistique cognitive sont analysées dans la première partie.

La seconde partie décrit l'émergence des thématiques et des positions caractéristiques de la linguistique cognitive. Quatre thématiques font l'objet d'un examen particulier : la notion de métaphore conceptuelle, la résurgence d'idées localistes, les questions liées à la théorie du prototype et à la catégorisation, et, pour finir, le concept de cadre (frame).

En conclusion, on souligne que la linguiste cognitive doit son unité au fait d'avoir construit autour d'un noyau central empiriste un réseau de thématiques et de positions théoriques interconnectées.

\section{Mots clefs}

Histoire de la linguistique, grammaire transformationnelle, sémantique générative, linguistique cognitive, métaphore, prototype, localisme, cadre

\section{Abstract}

This paper describes the birth of this theoretical conglomerate known as cognitive linguistics. In today's parlance, "cognitive linguistics" refers to a movement that was born in the United Sates in the 1970s and progressively took shape in the work of linguists who wished to break away from syntaxcentered tranformational grammar.

This split was to a large extent prepared by the movement of generative semantics, which had steered a number of linguists in a more semantically oriented direction. Generative semantics is given its due in the first part of the paper, in which I analyze the motivations of this movement, and the ways in which it may have prepared the ground for cognitive linguistics. The second part recounts how topics and positions characteristic of cognitive linguistics have emerged and gained momentum. Four central themes are given special emphasis: the notion of conceptual metaphor, the revival of localist ideas, questions related to prototype theory and categorization, and, finally, the concept of frame.

In conclusion, it is pointed out that cognitive linguistics gained its unity by weaving together themes and theoretical views of various origins around an empiricist core.

Keywords

History of linguistics, transformational grammar, generative semantics, cognitive linguistics, metaphore, prototype, localism, frame

\section{INTRODUCTION}

Dans cet article, je me propose d'explorer la période durant laquelle les protagonistes de la future linguistique cognitive américaine sont passés de la grammaire transformationnelle à des approches qu'on réunit aujourd'hui sous le nom de linguistique cognitive. 
Durant cette transition, le mouvement de la sémantique générative revêt une grande importance : les pères fondateurs de la linguistique cognitive américaine en étaient des partisans ou ont adopté à un moment donné des positions qui en étaient proches. Lakoff a revendiqué cette généalogie, d'autres, comme Langacker, la minorent. ${ }^{1}$

La caractéristique majeure de la sémantique générative est d'être une théorie transformationnelle qui met la sémantique au centre de la linguistique. Ce mouvement, comme nous le verrons, attira à lui des approches qui souhaitaient intégrer la sémantique à un cadre structuraliste. Elle fit aussi de la grammaire générative un cadre théorique acceptable pour des linguistes qui, comme Langacker, Talmy, et encore Chafe travaillaient sur l'anglais mais aussi sur des langues auxquelles la syntaxe générative de l'époque semblait inadaptée. Plus fondamentalement, la sémantique générative séduisit des linguistes qui mettaient au centre de la linguistique la question de la sémiose (de la mise en signes de la « pensée ») et étaient donc moins distributionnalistes que les générativistes plus proches de Chomsky.

Nous verrons que la linguistique cognitive a réemployé des matériaux légués par la sémantique générative et les approches connexes. Pour comprendre ce moment crucial de divergence au sein du générativisme, il nous faudra revenir sur les origines de la la sémantique générative et sur la période de transition qui suit son reflux. La première partie sera consacrée à cette tâche.

Le schisme provoqué par la sémantique générative n'explique pas tout. La linguistique cognitive s'est constituée aussi par l'importance donnée à des thématiques plus ou moins nouvelles, soit que ces thématiques infléchissent dans un sens cognitif des questions abordées à l'époque générativiste, soit qu'elles réveillent d'anciennes problématiques (les tropes, le localisme, la sémantique lexicale), soit, enfin, qu'elles introduisent des conceptions de facture récente (la théorie du prototype, les frames chez Fillmore). La légitimation de ces nouvelles thématiques s'est opérée en faisant apparaître des convergences et ces convergences ont contribué à associer les différentes approches en un complexe théorique qui peut se prévaloir d'une certaine unité de vues. La seconde partie sera consacrée à ces nouvelles thématiques.

Enfin, il me faut reconnaître les lacunes du présent article. Comme j'ai tenté de me situer aux alentours du point d'inflexion (approximativement, les années 1975-1980), les développements postérieurs, comme la montée des grammaires de construction et des traitements de corpus, seront très peu traités. Ces orientations sont aujourd'hui puissantes. En outre, les interactions entre la linguistique et les sciences cognitives, la psychologie des facultés employée en linguistique cognitive, certaines thématiques (comme la théorie des espaces mentaux et de l'intégration conceptuelle) ne seront pas abordées. Enfin, ma présentation de Langacker est succincte. Son évolution a fait l'objet d'une étude spécifique publiée dans cette même revue (Fortis 2010c). Le lecteur pourra s'y reporter pour de plus amples détails.

1 Lakoff $(1987$, p. 582) : «I view cognitive grammar as an updated version of generative semantics ». Langacker (1987, p. 4) : « Cognitive grammar is not in any significant way an outgrowth of generative semantics », même si, ajoute-t-il, « it does share with that conception a concern for dealing explicitly with meaning. ». 
Pour commencer, il nous faut revenir aux origines de la sémantique générative et à la brèche sémantique qu'elle a ouverte.

\section{LA SÉMANTIQUE GÉNÉRATIVE}

\subsection{Les structures profondes - le principe de Katz-Postal}

On sait que la représentation des homonymies de construction (du type the shooting of the hunters) est l'une des fins originellement poursuivies par la grammaire transformationnelle (Chomsky 1957). En outre, et depuis Harris, les transformations ont reçu la tâche inverse, qui est de représenter la relation qui unit des énoncés en relation paraphrastique mais distincts dans leur forme syntaxique, par exemple l'actif et le passif. Or, de l'idée qu'à un certain niveau d'analyse grammaticale les énoncés en relation paraphrastique ont la même représentation, et les homonymies de construction ont des représentations différentes, on passe aisément à l'idée qu'il existe un niveau où à toute identité de sens entre énoncés correspond une forme unique et à toute distinction de sens correspond une forme distincte. C'est ce niveau, que Katz et Postal (1964) caractérisent comme l'ensemble des indicateurs ou structures sous-jacents (underlying phrase markers), qui peut servir de base à l'interprétation sémantique.

Chez Katz et Postal (1964), les arguments en faveur de ces structures sousjacentes reflètent des préoccupations grammaticales et distributionnelles. Ainsi, les structures sous-jacentes doivent permettre de récupérer les relations grammaticales, de retrouver la structure en constituants, ou de rétablir les éléments effacés et permutés (Katz \& Postal 1964, p. 33s). En outre (et c'est là un argument distributionnel), ils considèrent que les énoncés en relation paraphrastique doivent avoir le même indicateur sous-jacent parce que ces énoncés ont les mêmes restrictions de sélection. Par exemple, ils attribuent à John's flying of the plane et the way in which John flies the plane le même indicateur sous-jacent au motif que les deux énoncés sont paraphrastiques et ont les mêmes restrictions de sélection, en particulier peuvent s'associer aux mêmes adjectifs, comme erratic ou foolish : John's flying of the plane is erratic / foolish etc. et the way in which John flies the plane is erratic / foolish etc. (Katz \& Postal 1964, p. 140).

Les structures sous-jacentes tendent ainsi à contenir tout ce qui est nécessaire à l'interprétation sémantique. Comme elles sont antérieures aux transformations, il s'ensuit que les transformations n'affectent pas ce qui permet d'interpréter un énoncé. Telle est la teneur du principe de Katz-Postal. ${ }^{2}$

Mais comment maintenir ce principe, alors qu'il existe manifestement des transformations qui altèrent le sens ? Il est évident, par exemple, que la transformation interrogative qui fait passer de 1 à 2 a affecte le sens de l'énoncé 1.

2 Ce principe avait été anticipé par Harris. On a « l'impression immédiate », disait Harris, que les transformations laissent le sens inchangé (Harris 1957, p. 339). 
(1) You will go home.

(2)a Will you go home?

b $\mathbf{Q}$ wh yes/no you will go home?

c I request that you answer whether yes or no you will go home.

Pour conserver aux transformations leur inocuité sémantique, Katz et Postal postulent des marqueurs abstraits qui déclenchent au niveau sous-jacent les transformations du type de la mise en fome interrogative. Dans l'exemple, ces éléments abstraits sont $\mathbf{Q}$, le marqueur d'interrogation, et $\mathbf{w h}$, le marqueur indiquant la portée de l'interrogation (Katz \& Postal 1964, p. 74-120). En 2b, l'interrogation porte sur «l'adverbe de phrase » profond « either yes or no ». La paraphrase de $2 \mathrm{~b}$ est donnée en $2 \mathrm{c}$.

Le principe de Katz-Postal a ainsi pour effet collatéral de conduire à un enrichissement des structures sous-jacentes. Lorsque Langacker, qui prenait pour cadre la théorie de Katz et Postal dans ses premiers travaux, abandonnera les marqueurs abstraits au profit de leur contenu sémantique, la transition vers la sémantique générative s'opérera d'elle-même (Fortis 2010c).

L'influence de Katz et Postal a été importante. C'est leur niveau des structures sous-jacentes que Chomsky va adapter sous le nom de structure profonde, apparemment sous l'influence directe de Postal (selon Jackendoff, cf. Huck \& Goldsmith 1995, p. 98-99 ; sur cette évolution vers la structure profonde, voir Harris 1993, p. 82-96). Bien plus, Aspects amorce une « sémantisation» des structures profondes, puisque Chomsky leur adjoint des restrictions de sélection contenant des marqueurs (+/- ANIME, +/- HUMAIN etc.) que Katz et Fodor avaient attribués à leur module sémantique (cf. Fodor 1977, p. 97, sur cette tension entre les deux théories).

\section{2. Émergence de la sémantique générative}

Parallèlement aux recherches de Katz et Potal, mais aussi sous leur influence, d'autres linguistes se dirigent vers une approche sémantique des structures profondes. C'est, semble-t-il, chez Lakoff, dans un memorandum daté de 1963 mais publié beacoup plus tard (1976), que cette approche reçoit le nom de sémantique générative.

\subsubsection{Les débuts de Lakoff}

La carrière de Lakoff débute par son mémoire d'undergraduate à l'université d'Indiana, qu'il rédige en 1961 (1972a [1961] ; Harris 1993, p. 103 ; Ruiz de Mendoza Ibáñez 1997). Lakoff y développe ce qui est apparemment la première story grammar, en prenant pour matériau la Morphologie du Conte de Propp. L'idée était de partir d'une structure de récit pour arriver à des formes de surface en utilisant des règles de réécriture. Le fait que les structures sous-jacentes soient exprimées en termes sémantiques permet de comprendre que Lakoff fasse de cette étude le levée de rideau de la sémantique générative. ${ }^{3}$ Lakoff part ensuite pour Cambridge (Mass.), au M.I.T., afin de collaborer au projet de traduction automatique dirigé par Victor Yngve. Il fait la connaissance de Ross, de McCawley,

3 «Generative semantics began with my undergraduate thesis in English literature at MIT. The thesis was the first generative story grammar » (Huck \& Goldsmith 1995, p. 107). 
et de Katz et Postal, qui travaillaient à cette époque sur leur Integrated theory (Katz \& Postal 1964). Selon Ross (Huck \& Goldsmith 1995, p. 122), l'influence que Postal exerce sur Lakoff est, à ce moment de sa carrière, très forte. C'est d'ailleurs sous la supervision de Postal que Lakoff termine la thèse qu'il avait entamée à l'université d'Indiana (Lakoff 1970 [1965]).

Dans le memorandum de 1963, Lakoff étend la notion de paraphrase à des énoncés dont les stuctures syntaxiques sont différentes (I fear John / John scares $m e$ ), et observe, à l'inverse, que des énoncés de structures syntaxique semblables peuvent être distingués par divers tests distributionnels (par ex. X hit a ball / hit a smash). Ces tests concernent en particulier la construction transitive, dont Lakoff montre qu'elle est compatible avec des verbes ou groupes verbaux qui réagissent différemment aux tests. Il en tire deux conclusions : il faut nécessairement enrichir la représentation sémantique des verbes et les restrictions de sélection, et, plus radicalement, l'analyse purement syntaxique est trop pauvre pour servir de cadre aux processus interprétatifs (Lakoff (1976) [1963], p. 50). La tâche du linguiste sera de dériver les formes à partir de ce que le locuteur veut dire, et que Lakoff appelle sa «pensée » (thought).

Pour diverses raisons, la thèse de Lakoff (1970 [1965]) poursuit cette évolution vers des structures sémantiques éloignées des formes de surface. Les structures sous-jacentes deviennent d'autant plus abstraites que Lakoff tend, comme Postal, vers une réduction des catégories lexicales (un appendice est consacré à la défense d'une super-catégorie VERBE englobant les verbes et les adjectifs). Si l'objectif premier de cette thèse est de formaliser les différents types d'irrégularité, la discussion s'ouvre aussi à l'analyse sémantique, et même à la pragmatique (figure en effet une représentation du focus informationnel). L'irrégularité conduit à s'intéresser au lexique, et le lexique à l'analyse sémantique, orientation que renforce l'extension considérable de la notion de paraphrase.

2.2.2. L'enrichissement des structures profondes : représentation logique L'une des caractéristiques de la linguistique cognitive américaine est son opposition à la formalisation logique. Comme la sémantique générative s'est approprié un formalisme logique (essentiellement la logique des prédicats du premier ordre), il est légitime de se demander si l'anti-logicisme de la linguistique cognitive reflète l'échec de cette appropriation. Je pense que certaines apories où conduisait la « logique naturelle » de Lakoff peuvent en effet expliquer ce rejet de la logique. Nous en verrons ci-après une illustration. Toutefois, il est vraisemblable que ces apories ont joué un rôle mineur dans l'anti-logicisme de la linguistique cognitive. D'autres facteurs, sur lesquels je reviendrai, ont été plus décisifs.

Afin de comprendre le statut de la logique dans la sémantique générative, il est bon d'évoquer les raisons qui ont motivé son introduction.

Ces raisons sont de trois ordres : 1) le formalisme logique permettait de résoudre certaines questions techniques que la grammaire transformationnelle ne pouvait traiter ou décrivait de manière inélégante ; 2) la réduction des catégories lexicales aux noms, verbes et phrases les rendait assimilables aux prédicats, arguments, et propositions de la logique des prédicats ; 3 ) étant donné le contexte culturel auquel appartenaient les sémanticiens générativistes, la logique apparaissait comme la seule option technique sérieuse. 
Je passerai rapidement en revue ces trois types de raisons.

Chez McCawley, l'introduction de variables indicées et de quantificateurs était motivée par des questions techniques relatives (entre autres) aux phrases en respective(ly) (du type x1, x2, x3... are respectively P1, P2, P3..., cf. McCawley 1976 [1968a]), à certains énoncés contenant des quantificateurs, et aux phrases dites de Bach-Peters. ${ }^{4}$ La portée du quantificateur sur des variables indicées permettait de traiter les ambiguiités référentielles, réintroduites en philosophie contemporaine par Quine (question de l'interprétation de dicto et de re ; McCawley 1976 [1970]). Ces questions d'ambiguïté référentielle, abordées d'abord par les sémanticiens générativistes puis par Jackendoff (1975), furent d'ailleurs à l'origine de la théorie des espaces mentaux (initialement appelés « espaces référentiels » par Fauconnier 1978 ; Fauconnier 1984), théorie qui est aujourd'hui considérée comme une branche de la linguistique cognitive. ${ }^{5}$

En même temps que McCawley introduisait des quantificateurs et des variables, plusieurs travaux s'accordaient pour réduire le nombre des catégories lexicales. Lakoff (1970 [1965]) proposait dans sa thèse une super-catégorie VERBE, englobant les verbes et les adjectifs, en se fondant d'ailleurs sur la synonymie supposée de I know / I am cognizant of, ou la présence dans les deux catégories d'une dichotomie statif / non-statif permettant de mettre en parallèle, pour les

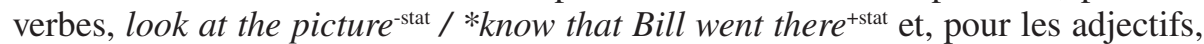
don't be noisy-stat / *don't be tall ${ }^{\text {stat }}{ }^{6}$ Ross (1969) proposait d'abolir, au niveau des structures sous-jacentes, la différence entre auxiliaires et verbes, et de traiter par exemple may dans rain may be good for the hair comme un prédicat profond (analogue à être possible). Lakoff (1970 [1965] et 1971) suggérait de détacher les quantificateurs (comme many ou two) des noms qu'ils modifient et d'en faire des prédicats, afin de leur donner dans un arbre syntagmatique une position qui corresponde à leur portée.

Enfin, la logique offrait une perspective à des linguistes que leur culture et la préoccupation d'articuler syntaxe et signification laissaient dépourvus d'outils d'analyse sémantique. Lakoff en fait foi, quand il remarque que « at that time, semantics meant logic - there was no other technically viable approach to semantics » (Huck \& Goldsmith 1995, p. 107).

4 Il s'agit de phrases à anaphores «croisées », par exemple (McCawley 1976 [1970], p. 144-145) : a boy who saw her kissed a girl who knew him. Dans une approche (typiquement transformationnelle) où le pronom est dérivé d'une copie de son antécédent, her correspondrait à a girl who knew a boy who saw her. L'antécédent contenant à nouveau her, l'analyse mènerait à une régression vicieuse. En analysant la phrase avec des variables indicées $\left(\mathrm{x}^{1}\right.$ saw $\mathrm{x}^{2}, \mathrm{x}^{1}=a$ boy who saw $\mathrm{x}^{2}, \mathrm{x}^{2}=a$ girl who knew $\left.\mathrm{x}^{1}\right)$, le problème disparaît.

Quant aux énoncés à quantificateurs, ce sont notamment les phrases du type everyone wants to win, qui n'est pas équivalente sémantiquement à la phrase dont elle serait issue par effacement du second everyone dans everyone wants everyone to win (transformation dite d'Equi-NP deletion). McCawley propose pour everyone wants everyone to win deux représentations logiques distinctes, dont l'une seulement permet l'Equi-NP deletion (McCawley 1976 [1970]).

5 Pour une présentation de cette théorie, voir Col (2010).

6 Newmeyer mentionne que l'incorporation des adjectifs à la catégorie Verbe avait été également défendue par Postal en 1965 (Newmeyer 1986, p. 82). 
Dans ce contexte, l'espoir suscité chez certains par la grammaire logique de Montague est d'autant plus compréhensible que sa diffusion chez les linguistes et sa jonction avec la grammaire transformationnelle s'effectuent à la fin de la sémantique générative (par exemple, Partee 1975 ; Partee 2011, pour un point de vue rétrospectif).

2.2.3. La sémantique générative entre logique et sémiose

En incorporant aux structures sous-jacentes des représentations logiques, la sémantique générative est confrontée à une double tâche : la logique est censé définir des lois de la pensée et fonder ainsi les relations entre énoncés sur des rapports d'équivalence, implication etc., tandis que la linguistique transformationnelle fonde en partie ces rapports d'équivalence, qui définissent les énoncés en relation de paraphrase, sur des restrictions de sélection. D'une part, nous devons avoir la capacité de penser logiquement, c'est-à-dire conformément aux règles d'inférence et aux lois logiques. D'autre part, on est obligé de constater qu'une langue particulière ne traite pas des énoncés logiquement équivalents de la même manière. Un exemple de Lakoff (1970) suffira à illustrer le problème.

Du point de vue logique, $(\mathrm{x})(\neg($ Sam saw $\mathrm{x}))$ est équivalent à $\neg \exists(\mathrm{x})$ (Sam saw $\mathrm{x})$, et les deux formules peuvent être prises pour des représentations logiques de Sam didn't see anyone. À son tour, Sam didn't see anyone est équivalent à Sam saw no one. Il serait raisonnable de conclure que les deux formules logiques $(\mathrm{x})(\neg$ (Sam saw x)) et $\neg \exists(\mathrm{x})$ (Sam saw x) peuvent indifféremment représenter chacune de ces deux phrases. Pourtant, absolutely n'est compatible qu'avec l'une d'elles (Sam saw absolutely no one mais *Sam didn't see absolutely anyone). Il y a par ailleurs des raisons de penser qu'absolutely exige la présence d'un quantificateur universel et que l'inacceptabilité de *Sam didn't see absolutely anyone est due à l'absence d'un quantificateur universel. Il s'ensuit que *Sam didn't see anyone ne peut être représenté par $(\mathrm{x})(\neg($ Sam saw $\mathrm{x}))$. Les équivalences logiques entre énoncés n'entraînent donc pas l'identité des structures sous-jacentes de ces énoncés. La synonymie entre énoncés est soumise à des contraintes non-logiques, difficiles à formaliser, et qui appellent une théorie, susceptible de rendre compte $\mathrm{du}$ fait qu'absolutely est en cooccurrence avec necessary, impossible, fascinating mais non avec possible, interesting etc. Dans l'explication par R. Lakoff de ces cooccurrences (Lakoff 1970, p. 237), la notion de valeur intense sur une échelle, chère à la linguistique cognitive, apparaît déjà. Une théorie plus souple et plus « cognitive » se profile. Et le soupçon point que la synonymie entre énoncés, qui permettait de leur attribuer une même structure sous-jacente, n'est pas un critère clair.

\subsection{L’influence de la sémantique générative}

La sémantique générative semble avoir été considérée, pendant quelques années, comme la théorie à la pointe de la recherche linguistique américaine (Seuren 1998, p. 503). Les raisons de cet attrait sont diverses. D'une part, le modèle des structures profondes sémantiques proposait une architecture d'une élégante simplicité, tout en ouvrant de nombreuses voies de recherche (perfomatifs, quantifications, ambiguïté référentielle, topicalité et focus, liaison entre actance et relations grammaticales 
etc.). D'autre part, la réintégration de la sémantique dans la linguistique américaine était en marche chez des non-générativistes, et cette réévaluation de la sémantique pouvait être récupérée par la sémantique générative. En outre, la grammaire générative syntactocentrée de l'époque était mal adaptée au traitement de problèmes posés notamment par des langues amérindiennes.

Dans les pages qui suivent, je traiterai de ces derniers aspects.

2.3.1 La réintégration de la sémantique et sa captation générative

Dans les années 1960, entre autres facteurs, les acquis de l'analyse componentielle en provenance de l'anthropologie (par ex. Goodenough 1965) semblent avoir encouragé des auteurs à développer le structuralisme américain en direction de la sémantique. Leurs tentatives étaient favorisées par l'évolution de la notion de morphème, qui était devenu chez Hockett une unité abstraite représentée par une forme (sur cette évolution, voir Matthews 1993, p. 76).

C'est ainsi que Sydney Lamb et Wallace Chafe, tous deux actifs avant que la grammaire générative ne devienne hégémonique, posent les bases de théories sémantiques dans le sillage du structuralisme. Lamb $(1964,1966)$ formule une théorie stratale des niveaux d'analyse qui est couronnée par une lexémique (lexemic stratum, plan des morphèmes comme unités de sens) et une sémémique (sememic stratum, plan des unités de sens, indépendamment de leur réalisation). Quant à Chafe $(1962,1965)$ il esquisse une « sémologie » (semology) inspirée de l'analyse componentielle des termes de parenté en anthropologie. Tous deux s'autorisent de la distinction hjelmslevienne entre la substance du contenu et la forme du contenu, considérée comme la structuration du contenu par la langue (Chafe 1967, Lamb 1964), et disent proposer une théorie de la forme du contenu, la substance du contenu ouvrant à l'expérience et à la cognition humaine.

Dans les années qui suivent, Lamb poursuivra le développement de sa théorie stratale, qu'il baptisera cognitive linguistics (à ma connaissance, la première théorie à porter ce nom), aujourd'hui renommée neurocognitive linguistics. Chafe, en revanche, adoptera la grammaire générative pour mettre en ouvre la sémantique qu'il avait d'abord esquissée dans un cadre structuraliste. Son propre modèle sera élaboré à une date précoce (les fondements en seront posés dès 1966 ; Chafe 1974). En 1970, il livrera au public une théorie aboutie, qui influencera vraisemblablement Langacker (Chafe 1970b, Fortis 2010c). Bien que Chafe n'épargne pas ses critiques à la sémantique générative, à laquelle il reproche sa conception laxiste de la synonymie, sa théorie en adopte néanmoins le principe fondamental, celui d'une génération des formes de surface à partir de structures sémantiques (Fortis 2012). Preuve supplémentaire de cette influence de la sémantique générative, la monographie que Chafe a consacrée à l'onondaga (une langue iroquoienne ; Chafe 1970b) était initialement intitulée, nous apprend-il (Chafe 2002), A Generative Semantic Sketch of Onondaga. Par la suite, sa propre version de la sémantique générative conduira Chafe à s'intéresser à la projection des rôles actanciels sur les relations grammaticales, à la structure informationnelle et à son expression, puis à se tourner vers une approche cognitive des relations grammaticales (Fortis 2012). Comme d'autres, au cours des années 1970, il fait entrer les facultés psychologiques (par ex. la mémoire ; Chafe 1973) dans ses descriptions avant de se consacrer à l'organisation du discours en unités régies par des contraintes cognitives et communicatives (Chafe 1979). 


\subsubsection{Universalisme et langues amérindiennes}

À l'instar de Chafe, deux protagonistes de la future linguistique cognitive, Langacker et Talmy, ont une activité d'amérindianiste. Tous sont influencés par l'universalisme chomskyen et considèrent pertinent de chercher un niveau de représentation où les langues sont identiques. Ainsi Chafe peut-il affirmer que «...the semantic structure of Onondaga differs from that of English in relatively trivial ways, and that the striking differences between the two languages arise largely as the result of postsemantic processes, which lead to markedly different surface structures $\gg$ (Chafe 1970a, p. 268).

De même, Talmy élabore son propre modèle de sémantique générative dans le cadre d'une comparaison entre l'anglais et l'atsugewi, guidé par l'idée qu'une structure profonde commune les unit (nous reviendrons sur la théorie de Talmy). Quant à Langacker, il se met en quête d'une théorie générale du passif qui (entre autres considérations) soit applicable à l'anglais et aux langues utoaztèques (Langacker \& Munro 1975) ; par ailleurs, cette théorie est une étape vers un modèle stratal de la proposition, encore proche des représentations de la sémantique générative et dont des éléments seront repris dans la Space Grammar, première version de la Cognitive Grammar (Fortis 2010c).

Il paraît probable que ce passage à des structures profondes sémantiques a été favorisé par la difficulté d'appliquer la théorie syntaxique de l'époque à des langues pour lesquelles elle était mal adaptée. Chafe et Talmy étaient confrontés à des langues polysynthétiques, et Langacker devait rendre compte de passifs apparentés à des impersonnels ou à des nominalisations. Un niveau d'analyse sémantique permettait de s'éloigner davantage des divergences structurelles de surface, mais exigeait aussi que soit éclairci le rapport entre structure sémantique linguistique et structure conceptuelle, ou pensée non « conventionnalisée » par la langue. Chafe et Langacker (peut-être à sa suite) tendront à les distinguer (Chafe 1970a, Langacker 1976), alors que Talmy s'engagera dès sa thèse (1972) dans une approche favorisant une confusion entre ces plans.

2.3.4. Des divergences sur les buts de la linguistique

Une déclaration de McCawley est très révélatrice de la conception que se faisaient les sémanticiens générativistes de la grammaire transformationnelle : " Aspects brought semantics out of the closet. Here was finally a theory of grammar that not only incorporated semantics (albeit very programmatically) but indeed claimed that semantics was systematically related to syntax and the construction of syntactic analyses a matter of much more than just accounting for the distribution of morphemes » (McCawley 1976b, p. 6, dans Huck \& Goldsmith 1995, p. 18).

Ces propos rejoignent ceux de Langacker, qui fait allusion ici à la grammaire générative :

«In recent years, linguists have recognized that meaning and syntax are crucial to an understanding of language. [...] They have also recognized that language is basically a psychological phenomenon, one that cannot be studied fruitfully just by observing linguistic behavior. » (Langacker 1973 [1967], p. 10).

Ces déclarations montrent qu'aux yeux de leurs auteurs la grammaire transformationnelle est responsable de la brèche sémantique où va s'engouffrer 
la sémantique générative. De fait, nous avons vu Lakoff, dès son memorandum de 1963, assigner à la linguistique la tâche de décrire la sémiose, c'est-à-dire la mise en forme de la pensée.

Langacker illustre de façon complexe cette conception de la grammaire transformationnelle. Il a été formé dans le cadre transformationnaliste (son directeur de thèse était Robert Lees, premier doctorant de Chomsky) ${ }^{7}$, et a mené aussi une carrière d'amérindianiste. Ses premières publications sur l'interrogation sont influencées par Postal et sont plus préoccupées de jeux dérivationnels formels que de sémantique ; il en est de même pour ses analyses des structures possessives (Langacker 1965, 1968). La grammaire générative lui est redevable d'une contribution décisive à la notion de commande (1969). Mais Langacker évolue rapidement vers une conception fonctionnaliste des structures et des transformations, s'efforçant de justifier les règles de mouvement par des considérations touchant à la mise en relief de certains constituants de l'énoncé (Langacker 1974b). C'est ainsi que dans sa théorie des constructions interrogatives, il délaisse les marqueurs profonds à la Katz et Postal, au profit de paraphrases sémantiques profondes (Langacker 1974a). Enfin, après avoir adopté une approche purement transformationnelle des énoncés possessifs, il s'oriente progressivement vers une analyse sémantique de ces énoncés, et notamment des verbes 'être' et 'avoir' (Langacker 1975). Cette analyse vient s'intégrer à un modèle grammatical de plus en plus centré sur le verbe et la relation de dépendance des arguments au verbe (Langacker 1975, 1978). L'architecture de ce modèle est sémantique. Les quatre strates qui la constituent (pour simplifier : propositionnelle / aspectuelle / temporelle et modale / performative) seront d'ailleurs reprises dans la Space Grammar (Langacker 1978). Ainsi, on peut dire que la Space Grammar conclut une dérive qui a pour point de départ la grammaire transformationnelle et la sémantique générative (Fortis 2010c).

\section{LA LINGUISTIQUE COGNITIVE EN GESTATION (1975-1980)}

Le reflux de la sémantique générative est perceptible vers 1975. Les raisons en sont diverses, et ont été détaillées par Harris (1993). Signalons-les brièvement.

Les sémanticiens générativistes manquaient d'assise institutionnelle et formaient peu d'étudiants. De plus, leurs recherches voulaient trop embrasser et tendaient à se disperser (Lakoff, par exemple, changeait constamment de modèle grammatical). Certains linguistes qui avaient été gagnés à la logique poursuivaient sur cette voie mais en adoptant des théories à fondement logique (la logique de Montague pour Partee). Dans le camp adverse, la réplique s'organisait (par exemple sous la forme du programme de la théorie $\bar{x}$ ), et des éléments de la sémantique générative étaient absorbés dans la grammaire générative mainstream, parfois sans reconnaissance de dette.

Vers 1975, deux de nos protagonistes, Lakoff et Langacker, sont désaffiliés du mouvement génératif et cherchent une autre voie. Comment s'opère, chez ces deux linguistes, la transition vers la linguistique cognitive?

7 Merci à Jacqueline Léon de cette information sur Lees. 
Langacker, nous l'avons vu, a progressivement dérivé vers un modèle grammatical centré sur la dépendance (plus que sur la constituance). Il commence à combiner dans une nouvelle théorie des éléments qu'il a pu trouver chez Talmy (l'importance de l'asymétrie figure / fond) et Fillmore (la notion de frame). Il réaménage aussi sa théorie de la proposition et des modalités et donne au nouveau modèle (après 1978) le nom de Space Grammar, pour des raisons qui tiennent à la représentation spatiale des strates de la proposition et à une représentation localiste des modalités verbales (Langacker 1978). Entre autres considérations, son analyse gestaltiste du sens lexical le conduit à introduire des représentations diagrammatiques, qu'il associe à une faculté toute puissante d'imagerie (Langacker 1979, 1981). Cette faculté devient chez lui une façon de conceptualiser les objets et leurs relations en fonction des conventions sémantiques propres à une langue particulière. L'importance de l'imagerie vient aussi du fait que Langacker a éprouvé des doutes de plus en plus sérieux à l'égard des primitives sémantiques et des paraphrases employées en sémantique générative et dans les modèles apparentées (dont le sien ; Langacker 1976). Ces doutes ont pu être alimentés par les critiques que Chafe avait formulées contre la notion de synonymie usitée en linguistique générative (Chafe 1971). Ils ont sans doute été nourris aussi par les arguments que Bolinger venait de rassembler dans un ouvrage qui était une machine de guerre contre la synonymie des paraphrases en grammaire générative (Bolinger 1977).

En résumé, même si l'évolution de Langacker, très complexe, est une réélaboration constante, on peut discerner chez lui une période où il donne à ses travaux une inflexion cognitive, période qui correspond à peu près aux années 1975-78, et qui culmine avec la première version de la grammaire cognitive (la Space Grammar). Le nom de cognitive grammar s'impose à partir de 1986 (Langacker 1986), dans un article publié dans une revue de science cognitive, non de linguistique.

Quant à Lakoff, il traverse une période stérile de presque deux ans (1975-1977) qui fait suite à un Summer Institute organisé à Berkeley en 1975. Au cours de cette conférence, la convergence qu'il perçoit au sein de plusieurs approches semble annoncer pour lui une nouvelle configuration interdisciplinaire :

«Plusieurs exposés historiques ont été présentés cet été-là. D’abord, il y a eu le premier exposé par Eleanor Rosch de ses résultats sur les catégories de niveau de base. En second, il y a eu le premier exposé de Talmy sur ses travaux qui montraient que les concepts associés aux relations spatiales avaient des primitives de nature topologique et orientationnelle [ex. front / back]. En troisième lieu, Charles Fillmore, qui, pour la première fois, défendait la nécessité d'une approche sémantique en termes de cadre (frame). Et en quatrième, Paul Kay présentait son travail réalisé avec Chad MacDaniel sur le fondement neurobiologique de la catégorisation des couleurs » (Huck \& Goldsmith 1995, p. 117).

La nouvelle configuration que Lakoff pressent met en relation la psychologie (Rosch), la neurobiologie (MacDaniel) et la linguistique (Talmy et Fillmore) en articulant directement les catégories linguistiques à la cognition visuelle ou à la saisie de complexes expérientiels (chez Fillmore) : le niveau de base de catégorisation est issu d'un principe de ségrégation perceptive et motrice des objets, le modèle neurobiologique de MacDaniel associe directement couleurs focales et pics de réponse neuronales, les primitives topologiques de Talmy sont 
des principes de saisie des relations spatiales qui conditionnent leur expression linguistique, et les cadres de Fillmore (1975) paraissent refléter une priorité de ce qui est conjoint dans l'expérience sur ce qui en est abstrait.

Lakoff interprète apparemment cette articulation directe entre saisie perceptive, cerveau et expérience comme une remise en cause des codes intermédiaires que seraient les formalismes :

« Et donc, face à toutes ces preuves, durant cet été de 1975, je me suis rendu compte que la grammaire transformationnelle et la logique formelle étaient désespérément inadaptées et j'ai arrêté de faire de la sémantique générative. »(Ruiz De Mendoza Ibáñez 1997, p. 39).

Le revirement de Lakoff ne peut cependant se résumer à cette conférence. Nous avons vu qu'il devait affronter des problèmes théorique liés aux objectifs de la sémantique générative, qui devait articuler logique et contraintes linguistiques sur l'expression logique de la pensée. D'autre part, la réduction des formalismes à des processus psychologiques semblait impossible : comment accorder des définitions extensionnelles ou des mondes possibles avec l'impératif de plausibilité cognitive (Lakoff 1982, 1987) ? La question tourmentait aussi des linguistes qui allaient, malgré cette difficulté, s'engager dans la sémantique logique (Partee 1979). Enfin, d'autres apories, relatives à des constructions « déviantes », avaient surgi. ${ }^{8}$

Durant les deux années qui suivent, Lakoff rumine sur le changement théorique qu'il effectue par la publication en 1977 de Linguistic Gestalts.

L'article plaide pour une « linguistique expérientielle », c'est-à-dire ouverte au monde de l'expérience humaine. C'est le monde de l'expérience, souligne Lakoff, qui permet de comprendre les structures linguistiques, entre autres raisons parce que ces structures sous-déterminent les interprétations possibles, ou parce qu'elles sont soumises à des contraintes sémantiques qui renvoient à l'expérience humaine. Un exemple simple du premier type serait la série des noms composés topless dancer, topless bar et topless legislation, où les diverses interprétations sont sousdéterminées par la structure, qui est toujours la même. Un exemple du second type est fourni par les contraintes multiples susceptibles d'expliquer la projection des rôles actanciels sur les fonctions grammaticales. À titre d'illustration, Lakoff rend compte de la différence d'acceptabilité entre (3) et (4) par la notion de responsabilité (causale) primaire (Lakoff 1977, p. 248-249).

(3) Bean curd digests easily.

(4) * Bean curd eats easily.

$\mathrm{Si}$ (4) est inacceptable, c'est qu'une des conditions de projection sur la fonction de sujet exige que l'actant visé ait la plus grande part de responsabilité causale. Cette condition n'est pas satisfaite en (4), parce que manger requiert de l'agent, non du

8 Les constructions en question sont les « amalgames syntaxiques ». L'exemple suivant illustre le phénomène : John invited you'll never guess how many people to you can imagine what kind of a party, for God knows what reason on wasn't it last Saturday? (Ruiz De Mendoza Ibáñez 1997, p. 36 ; Lakoff 1974). Dans la période récente, le retour de la notion de construction comme intégration d'éléments qui ne sont pas nécessairement des constituants « classiques » a permis de traiter le problème à nouveaux frais (mais la question avait été soulevée par Bolinger 1961). 
patient, la plus grande implication causale. Ce supplément expérientiel qui permet de comprendre, construire, rejeter une structure linguistique compose avec cette structure ce que Lakoff appelle une gestalt.

Cette notion de gestalt est d'autant plus vague qu'elle sert à unifier une collection très disparate de phénomènes, que Lakoff puise dans la littérature générativiste ou anti- ou péri-générativiste, chez Gruber, Fillmore, Postal et d'autres : les noms composés et les expressions idiomatiques, objections usuelles aux règles compositionnelles, les phénomènes d'attraction entre constructions (chers à Bolinger), les cadres à la Fillmore, les métaphores, les relation entre rôles actanciels et relations grammaticales, nouvellement mises au premier plan chez Fillmore, dans la grammaire relationnelle de Postal et Perlmutter et chez Lakoff lui-même (Lakoff \& Thompson 1975). L'ensemble donne l'impression que la gestalt sert de principe fourre-tout à un énième programme théorique de Lakoff. La théorie des métaphores conceptuelles lui permettra de reprendre la main.

\section{L'ÉMERGENCE DES THÉMATIQUES DE LA LINGUISTIQUE COGNITIVE}

Il y a plusieurs manières possibles d'aborder la formation de la linguistique cognitive : on peut enchaîner les biographies intellectuelles et montrer comment les auteurs s'influencent et participent à un mouvement commun ; on peut aussi faire l'histoire des thématiques, des concepts et des outils théoriques. J'ai choisi cette seconde voie, afin de mettre en exergue les zones de convergence qui associent les voies de recherche empruntées, et qui contribuent à donner son unité à la linguistique cognitive. Dans la suite seront présentées quatre des thématiques principales de la nouvelle linguistique cognitive : les métaphores conceptuelles, le prototype, le localisme, et les cadres (frames).

\subsection{La théorie des métaphores conceptuelles}

Linguistic Gestalts suivait chez Lakoff une période de rumination coïncidant avec le reflux de la sémantique générative. Des aspects de la notion de gestalt vont être reconduits dans les réflexions futures de Lakoff, mais le virage pris à la suite de cet article n'en est pas moins déconcertant : pourquoi un linguiste nourri au sein de la grammaire générative a-t-il ressenti le besoin de se tourner vers l'étude des métaphores ?

Si l'on en croit Lakoff, ce changement de cap serait une découverte due aux circonstances (Ruiz de Mendoza Ibañez 1997) : un cours qu'il fait en 1978 sur des articles de Searle (dont celui paru en 1979) et Davidson (1978) sur la métaphore, une étudiante en émoi qui vient s'épancher dans ledit cours et prononce la métaphore que l'assistance s'empresse de filer (« mon petit ami a dit que notre relation était dans une impasse »), et la « découverte » qu'une métaphore filée implique tout un réseau de correspondances entre le domaine cible et sa description figurée ; enfin, la rencontre en 1979 avec le philosophe Mark Johnson, qui s'intéresse à l'esthétique et à la métaphore.

Le produit de ce revirement et de la collaboration avec Johnson est Metaphors we live by (1980), ouvrage qui sera l'un des best sellers de la linguistique cognitive 
(vendu à 9000 exemplaires dès la première année ; Lawler 19839) et suscitera un «Metaphernboom », pour reprendre Bamberg (1983).

Le style du livre est non technique, la nouveauté du sujet est claironnée, et les ambitions sont démesurées, puisqu'il ne s'agit de rien moins qu'une remise en cause d'un pan de la tradition philosophique occidentale et de sa théorie de la connaissance (disons pour simplifier le « rationalisme » et le « logicisme »). Pourtant, nous le verrons, le livre contient peu d'idées nouvelles.

4.1.1. Les thèses de Metaphors We Live By

L'idée fondamentale de Lakoff et Johnson est que la métaphore est un processus qui permet d'appréhender un domaine de l'expérience dans les termes d'un système de concepts propres à un autre domaine. Ce système confère au domaine cible ${ }^{10}$ une structure qui lui manque, permet de l'envisager sous une nouvelle perspective, ou de le mettre en relation avec d'autres systèmes. L'expérience du temps constitue un exemple de domaine « amorphe » structuré par un système métaphorique, en l'occurrence les relations spatiales (cf. in the weeks ahead of us, the time will come when...).

D'autres principes ou thèses complètent cette idée fondamentale :

Cohérence : les métaphores sont partiellement contraintes par un principe de cohérence. Ainsi, pour rester dans le domaine spatial, l'association de la direction verticale avec la joie, la santé et la valuation positive (I'm feeling up, my spirits rose) se renforcent l'une l'autre. Des systèmes sources hétérogènes peuvent communiquer entre eux par une « imagerie » commune. En fait foi l'exemple suivant (les sytèmes source sont indiqués en capitales ) :

So far [JOURNEY] we have constructed [BUILDING] the core [CONTENT] of our argument. La cohérence peut donc concerner plus d'un système source.

Primauté $d u$ physique : ce qui est non physique, ou « amorphe », est typiquement conçu en termes physiques. La notion de localisation dans un espace borné s'applique ainsi au temps (dans deux heures), à l'appartenance, il est dans la police, mais aussi à un état, he is in love).

Complexité des concepts fondamentaux : les concepts fondamentaux ne sont pas des primitives mais sont dotés d'une structure complexe. Par exemple, et à rebours de ce qui était communément posé en sémantique générative (quoique problématisé par Talmy 1972, 1976, et d'autres), il n'existe pas de primitive CAUSER, mais un complexe CAUSATION qui correspond à un scénario typique de causation (un agent humain effectuant par manipulation directe un changement d'état perceptible chez le patient).

Structure interne des concepts : comme nous venons de le voir à propos de la notion de causation, Lakoff et Johnson importent la théorie du prototype dans leur analyse des concepts. Je reviendrai sur la théorie du prototype ci-après.

9 Il est intéressant de noter que Lawler, en linguiste de l'époque, rattache le livre de Lakoff et Johnson à ce qui lui semble s'en rapprocher le plus, c'est-à-dire à la sémantique générative.

10 Je parle ici de système source (le comparans) et de système ou domaine cible (le comparandum), suivant l'usage postérieur de Lakoff et Johnson (1999). Dans Metaphors We Live By, Lakoff et Johnson n'emploient pas encore la terminologie source / target. 
Véridicité des métaphores : les métaphores étant susceptibles de constituer les conditions mêmes de l'appréhension d'un domaine, elles ne sont pas alors subordonnées à un sens littéral mais elles peuvent être vraies ou fausses. La notion de vérité elle-même doit être relativisée puisqu'un énoncé peut n'être évaluable qu'en présupposant un système métaphorique.

Véridicité $\neq$ vériconditionnalité. L'équivalence entre le sens et ce qui détermine les conditions de vérité d'un énoncé littéral est un mythe. Les discours scientifiques sont en partie construits sur des systèmes métaphoriques très stabilisés dont la vérité est gagée sur leur capacité à nous adapter à notre environnement naturel et social. Nous entrevoyons ici un thème fictionnaliste et pragmatiste, celui de la fiction utile et de l'utile comme vrai.

Origine «expérientielle » des métaphores : l'expérience du monde physique entraîne la formation de gestalts, d'associations récurrentes entre composants d'événements vécus. La causation prototypique en est une illustration (voir supra). Ces « gestalts expérientielles » forment le noyau des systèmes sources les plus fondamentaux. L'acolyte de Lakoff, Johnson a beaucoup développé cette idée, en lui annexant une notion de schéma qu'il voit comme un rejeton du schème kantien (Johnson 1987 ; Nyckees 2007 pour une critique vigoureuse de ces thèses).

4.1.2. Metaphors We Live By en contexte

Lakoff et Johnson ne font aucune allusion à l'histoire de la rhétorique, ni aux travaux contemporains sur la métaphore. Quoique Ricœur soit cité en préface, il l'est non pour La Métaphore Vive (1975, paru en anglais en 1978 sous le titre The Rule of Metaphor) mais pour ses réflexions sur la nature des sciences humaines.

Pourtant, il faudrait plusieurs pages pour présenter les théories qui ont fait de la métaphore une opération cognitive, un principe fondamental de fonctionnement du langage, et la raison de réexaminer la notion de vérité. Je m'abstiendrai de cette tâche avec d'autant moins de regret qu'elle a été menée à bien dans plusieurs travaux (Danesi 2001, Nerlich \& Clarke 2001, Burkhardt \& Nerlich 2010). Des parallèles avec Vico (1744), Mauthner (1923), Gerber (1871-1874), Nietzsche (1873), lui-même lecteur de Gerber, et d'autres ont été observés. Ces précurseurs sont ignorés de Lakoff et Johnson (1999) dans leur histoire " cognitive ${ }^{11}$ de la «pensée occidentale », un parcours de quelques grands penseurs qui va des présocratiques à Chomsky. Pour notre propos, la question qu'il convient de poser n'est pas de savoir qui sont les précurseurs, mais dans quel contexte a émergé la théorie de Lakoff et Johnson, quelles influences ils ont vraisemblablement subies, et pourquoi la métaphore était (re)devenue un sujet digne d'attention.

En milieu américain et dans les années 1970, on peut dire que se conjuguent plusieurs influences intellectuelles :

(1) La réflexion sur la relativité linguistique, surtout chez Whorf ;

(2) Les travaux de Richards (1936), Black (1955, 1962, 1979), et Ricœur (1975), qui commente d'ailleurs Richards et Black ;

11 Histoire cognitive au sens où les pensées philosophiques sont décrites par les métaphores conceptuelles qu'elles sont censées mettre en œuvre. 
(3) L'approche « pragmatique » d'interprétation des énoncés, illustrée en philosophie par Searle (1979) et en linguistique par la thèse de Nunberg (1978), que Lakoff a lue avant sa publication (comme nous l'apprennent les remerciements);

(4) L'analyse de la métaphore comme instrument heuristique et outil de modélisation d'un domaine. Schön invoque ainsi la métaphore comme principe de « déplacement des concepts » et outil heuristique face à une situation problématique, notion qu'il emprunte à Dewey, à qui il a consacré sa thèse (Schön 1963, 1979). La continuité entre perception et conceptualisation, et l'insistance sur le caractère adaptatif de la métaphore sont des thèmes pragmatistes. Notons que la proximité à Dewey ne sera revendiquée que bien plus tard par Johnson (2008). Enfin, Schön (1979), comme Lakoff après lui, explique qu'un système métaphorique peut être un instrument idéologique dans la mesure où il impose les termes d'un débat politique. Et pour désigner la perspective produite par un tel système, Schön emploie la notion de frame, cette fois en un sens qui renvoie apparemment à la théorie sociologique de Goffman (1974).

(5) Les recherches en psychologie, en particulier sur le développement de la capacité à comprendre les métaphores et les proverbes (Ortony et al. 1978 en offrent une synthèse). Signalons aussi la publication d'un important ouvrage sur la métaphore, dirigé par Ortony (1979; l'article de Schön y figure), et dont Lakoff a pris connaissance avant qu'il ne soit publié (comme nous en fait part Ortony luimême).

(6) Des études ponctuelles mais qui ont eu leur importance dans l'histoire de la linguistique cognitive. Je pense ici à celles de Reddy (1969, 1979, encore publié dans Ortony 1979), qui attaquaient le lien naïf entre sens littéral et réalité, réveillaient la problématique de la relativité linguistique en insistant sur le rôle de la métaphore d'usage ordinaire, et illustraient en détail l'étendue des ramifications d'un réseau métaphorique. En outre, chez Reddy (1979) ce réseau était qualifié de cadre (frame), terme apparemment pris à Schön, et par lequel Reddy voulait signifier qu'un réseau de métaphores implique une certaine façon de concevoir un domaine d'objets.

D'autres filiations sont envisageables mais beaucoup moins sûres, comme celle de la métaphore radicale (entendue comme équivocité des racines) et de son rapport au mythe, et dont les jalons seraient Max Müller, Cassirer et Susanne Langer (sa traductrice), à qui on pourrait ajouter, selon Nerlich et Clarke (2001) la rhétoricienne américaine Gertrude Buck (1871-1922).

Je m'attarderai quelque peu sur les influences de Whorf, Richards et Black, Nunberg et Searle.

4.1.3. Whorf

Lakoff semblant lire ou relire Whorf au moment où il écrit Linguistic Gestalts (1977), il n'est pas exclu que Metaphors We Live By contienne aussi des réminiscences whorfiennes.

Chez Whorf la «pensée habituelle » (habitual thought) est dépendante de schémas linguistiques dont certains au moins sont construits sur une armature métaphorique, au point que «we can hardly refer to the simplest nonspatial situation without constant resort to physical metaphors. I "grasp" the "thread" of another's 
arguments, but if its "level" is "over my head" my attention may "wander" and "lose touch" with the "drift" of it, so that when he "comes" to his "point" we differ "widely" » (Whorf 1956, p. 146). Comme le feront Lakoff et Johnson, Whorf associe la spatialisation du temps dans les cultures occidentales à une technologie et à un mode d'organisation sociale du temps, sans toutefois poser une dépendance de la culture à la langue (il parle d'une « consonance »; 1956, p. 154). La position de Lakoff et Johnson sur la relativité linguistique est difficile à cerner. Ils semblent bien faire dépendre la conceptualisation linguistique de corrélations expérientielles primitives, et attribuent en tout cas la thèse d'une telle dépendance à Whorf (Lakoff \& Johnson 1980, p. XI), ce qui me paraît être un contresens.

4.1.4. Richards et Black

Dans beaucoup d'études qui leur font suite, ce sont Richards et Black qui ont fixé les termes du débat : la métaphore est-elle une comparaison entre deux termes dont l'esprit met en rapport des similarités, ou bien une interaction créatrice de similarités ? L'alternative est reprise par Lakoff et Johnson (1980, p. 151s), qui la tranchent, comme Richards et Black, en faveur de la position interactionniste. Voyons de plus près ce que nos deux auteurs ont pu reprendre de Richards et Black, en commençant par Richards.

Tout d'abord, l'ouvrage de Richards (1936) se concentre essentiellement sur la métaphore, comme Lakoff et Johnson. ${ }^{12}$ En outre, de même que Lakoff et Johnson considèrent la métaphore comme une projection de la source sur une cible, de même Richards la définit par le complexe vehicle / tenor et leur interaction, et non pas seulement par le vehicle. Ensuite, Richards s'intéresse à l'usage ordinaire, pour la raison que la métaphore est un principe «vital » et omniprésent du langage. À l'instar de Lakoff et Johnson (1980, p. 190), Richards (1936, p. 48) lie la fiction du sens littéral à un discours « rigide », celui de la science. Pour Richards, le discours scientifique ne favorise la croyance en un apport sémantique indépendant des mots que pour la raison que les contextes y sont très contrôlés, alors que la règle commune est plutôt l'absence de signification hors contexte. Soulignons-le : le rôle du contexte est fondamental. Comme le dit Ricœur, pour Richards, « les mots n'ont pas de signification propre parce qu'ils n'ont pas de signification en propre » (Ricœur 1975, p. 101). Enfin, la métaphore imprègne la langue parce que la pensée elle-même est métaphorique (1936, p. 94).

Prolongeant la vision interactionniste de Richards, Black envisage la métaphore comme un « filtre » (1954) permettant d'envisager un domaine cible au prisme d'un système source. La métaphore opère en mettant en relation des complexes de représentations relevant de l'opinion commune ou de la doxa (Black 1954, 1962, 1979). Elle ne repose ni sur la perception d'une incongruité, ni sur celle de similarités qui seraient déjà présentes, mais constitue plutôt un «instrument

12 Pour être juste, il faut noter que Lakoff et Johnson mentionnent aussi la personnification, la métonymie et la synecdoque. Métonymie et synecdoque ont plutôt un rôle de référence indirecte (un thème mis en exergue en sémantique générative ; Borkin 1972), alors que la métaphore est véritablement créatrice de structure et de perspective. Cependant, la distinction métonymie / métaphore n'est pas toujours très claire : mouvement et espace sont ainsi qualifiés de «métaphores » du temps, alors que temps et espace sont des dimensions du mouvement (ce qui établirait plutôt entre eux un rapport métonymique). 
cognitif » permettant de voir des rapports qui ne préexistaient pas (Black 1979). On remarquera aussi que Black présente les métaphores sous forme prédicative («Man is a wolf»), comme Lakoff et Johnson (1980, 1999), et que cette forme signale la nature relationnelle de la métaphore.

4.1.5. La métaphore comme fonction pragmatique

Les années 1970 sont une période de floraison de la pragmatique : actes de parole, verbes performatifs, présuppositions, topicalité et focus informationnel, indexicalité sont autant de questions présentes dans les travaux générativistes, en linguistique fonctionnelle, chez des auteurs qui s'écartent de la syntaxe de la phrase (comme Chafe), ou même en psycholinguistique. Ariel (2010, p. 4) parle ainsi d'un pragmatic turn qu'elle situe au début des années 1970.

Le projet qui sous-tend l'approche pragmatique de la métaphore est la formulation de procédures permettant de dériver en contexte l'interprétation visée par le locuteur. Searle (1979) établit ainsi un parallèle entre l'acte de parole indirect (Peux-tu me passer le sel ?) et la métaphore, et hasarde quelques principes (très vagues) permettant de circonscrire l'interprétation finale. Il s'oppose par ailleurs à la thèse interactionniste.

Beaucoup plus fouillée est l'étude de Nunberg (1978), qui envisage la métaphore comme un cas particulier d'indétermination de l'interprétation. L'originalité de Nunberg est de formuler des règles d'interprétation en prenant pour point de départ ce qu'il est convenu d'appeler la référence ostensive indirecte (ou deferred ostension, la notion vient de Quine), du type Hearst bought that, où that pointe vers un exemplaire de journal mais réfère en réalité au journal comme société commerciale. Dans l'approche de Nunberg, la faible teneur sémantique du déictique révèle par contraste l'ampleur des procédures pragmatiques à l'œuvre. Ces procédures sont ensuite appliquées aux nominaux. En fin de compte, référence indirecte et métaphore exigent des opérations similaires et relèvent d'un fonctionnement général du langage, où interviennent nécessairement des systèmes de croyances rationnelles déterminées par le contexte. Nunberg montre ainsi que la sémantique est continue avec la pragmatique et que la métaphore n'est pas un phénomène marginal. Toutefois, il achoppe sur la motivation des métaphores, que Lakoff et Johnson chercheront dans les « gestalts expérientielles » ou les schèmes.

Signalons enfin que Nunberg prolongeait des études de sémantique générative qui montraient la difficulté de formuler clairement des critères permettant de déterminer la référence d'un nominal. Or, de tels critères étaient importants pour la syntaxe transformationnelle, par exemple pour le traitement des anaphores ou des réflexifs (cf. Borkin 1972). De tels problèmes ont eux aussi préparé les esprits aux questions de référence indirecte, et donc à la thématique des figures (en l'occurrence la métonymie).

\subsection{Le localisme}

Parmi les exemples qui illustrent la structuration d'un domaine par un système métaphorique figure le champ des métaphores spatiales du temps. De fait, les relations spatiales jouent en sémantique cognitive un rôle privilégié, dont le retour du localisme est un des symptômes. À une date plus récente, la convergence entre sémantique cognitive et études diachroniques a mis en exergue l'importance des marqueurs spatiaux dans la grammaticalisation (notamment à partir des travaux de 
Bernd Heine et de ses collaborateurs, Heine, Claudi \& Hünnemeyer 1991). Enfin, les linguistes cognitivistes font un large usage de représentations schématiques, de « diagrammes », dont la réalité psychologique est difficile à déterminer mais qui semblent souligner le lien entre perception et concepts linguistiques. S'est ainsi constituée une thématique localiste en sémantique lexicale, en théorie de la diachronie et au niveau de la représentation même des structures linguistiques.

Empruntant à Lyons sa définition, nous dirons que le localisme est :

«The hypothesis that spatial expressions are more basic, grammatically and semantically, than various kinds of non spatial expressions [...]. Spatial expressions are linguistically more basic, according to the localists, in that they serve as structural templates, as it were, for other expressions; and the reason why this should be so, it is plausibly suggested by psychologists, is that spatial organization is of central importance in human cognition. " (Lyons 1977, p. 718).

Le localisme n'est pas une idée nouvelle (Parret 1989, Fortis, à par.). Il a été débattu au cours du XIX ${ }^{\mathrm{e}}$ siècle en théorie des cas, dans des contextes divers, liés d'abord à une sémantique post-kantienne, puis au psychologisme (chez Wundt). Hjelmslev (1934-5) proposa une théorie néo-localiste des cas, qu'il introduisit par une synthèse des travaux précédents. Au début des années 1970, la tradition localiste d'analyse des cas est revitalisée par Anderson, qui marie le localisme ancien à une approche formaliste, de type génératif (Anderson 1971).

4.2.1. Le localisme en linguistique cognitive

Le localisme de la linguistique cognitive ne paraît pas puiser à cette source historique (bien qu'Anderson ait été lu par Langacker et d'autres peut-être). La réémergence du localisme en linguistique cognitive conjugue, semble-t-il, deux mouvements : un localisme "générativiste », qui identifie les rôles actanciels fondamentaux à des relations spatiales, ou modèle les structures profondes sur la représentation des relations spatiales (Gruber 1965, Talmy 1972) ; et, second mouvement, une série d'études sur les déterminants cognitifs de la lexicalisation des relations spatiales, qui sont dues à des psychologues comme Clark, Miller et Johnson-Laird (Clark 1973, Miller \& Johnson-Laird 1976). Un peu plus tard, des travaux élaborés dans l'optique d'une formalisation des procédures d'emploi des prépositions spatiales (plutôt dans la lignée de Miller et Johnson-Laird) ont convergé avec ceux de linguistes formés à la linguistique cognitive naissante (Herskovits 1986 pour les premiers, Vandeloise 1986 pour les seconds).

Nous sommes donc, semble-t-il, en présence de deux traditions localistes relativement disjointes, l'une ancienne et ayant pour objet les cas, l'autre, récente, née dans le milieu générativiste et en psychologie. En outre, l'une de ces traditions est partiellement initiée par deux travaux qui sont des objets linguistiques non identifiés et ne font référence à aucune étude antérieure. Je veux parler ici des thèses de Gruber (1965) et Talmy (1972).

4.2.2. Le localisme "générativiste »: Gruber

La thèse de Gruber (1965) est une tentative de décrire les rôles actanciels, la valence et le marquage des compléments du verbe au moyen de représentations profondes des verbes. Ces représentations, dites «prélexicales », incorporent des 
prépositions profondes qui identifient les rôles actanciels. Le sujet de buy est par exemple marqué FROM au niveau prélexical, c'est-à-dire est identifié comme actant source du transfert du bien vendu. L'incorporation sert aussi à représenter les constructions alternantes d'un même verbe : un verbe qui a plusieurs constructions (par ex. climb $(G N)$ / climb up $(G N)$ ) est décrit comme incorporant facultativement une préposition (ou un adverbe).

Gruber observe que les prépositions incorporées qui identifient des rôles spatiaux servent aussi à l'expression d'autres relations. Ces relations relèvent de quatre domaines « ontologiques »: l'identité des référents (domaine « identificationnel »), la possession (domaine « possessionnel »), les propriétés (domaine « classificatoire » ou de class-membership) et la communication (ou « transfert abstrait»). Les exemples suivants illustrent la similitude des structures qui lexicalisent des relations de ces différents domaines : the coach turned into a pumpkin ( identificational »), John gave a book to Bill («possessional »), John translated the letter from Russian to English ( class membership »), John reported to Mary that («abstract transferred entity »)... (1965, p. 47s).

Si Gruber ne se déclare pas localiste («there is no particular priority intended for the sense of concrete motion », ibid., p. 48), en pratique ses analyses modèlent les rôles actanciels sur les relations spatiales. Il appelle d'ailleurs abstract motion la généralisation de ces relations aux rôles actanciels de verbes non spatiaux.

L'influence de Gruber est perceptible chez Fillmore, qui essaie d'analyser par d'autres moyens les alternances de construction que Gruber avait décrites grâce à sa notion d'incorporation. Il pourrait aussi lui avoir emprunté ses cas Source et But. Gruber a inspiré à Jackendoff (1983) sa théorie localiste des fonctions sémantiques primitives. Quant à Talmy, il est difficile de savoir si Gruber l'a influencé directement, puisqu'il ne le cite pas dans sa thèse. On peut noter toutefois une proximité certaine entre l'analyse par Gruber du rôle actanciel de thème et la notion de figure chez Talmy.

Il est temps désormais de nous tourner vers ce dernier et de résumer sa théorie initiale, qu'il présente dans sa thèse de 1972.

4.2.3. Le localisme « générativiste » : Talmy

Comme la thèse de Gruber, celle de Talmy s'inscrit dans le générativisme. Comme la thèse de Gruber, elle fait figure d'hapax théorique, non seulement par les idées qui y sont défendues, mais aussi parce qu'elle est dépourvue de références, et use d'une terminologie inhabituelle, voire baroque. Cependant, elle est clairement affiliée à la sémantique générative : elle postule des structures profondes sémantiques, des transformations pré-lexicales, et emploie des arbres de dérivation.

À l'instar de Chafe, Talmy cherche un niveau de représentation sémantique qui puisse servir d'étalon de comparaison entre une langue polysynthétique (l'atsugewi) et l'anglais. Sa stratégie est de choisir un type de situation, la localisation spatiale statique et dynamique, et de comparer les moyens lexicogrammaticaux mis en œuvre par chaque langue pour l'exprimer. Ce sont ces moyens lexicogrammaticaux qui distinguent les langues. À un niveau profond, elles sont identiques, c'est-à-dire structurent la situation de la même manière. Cette structure commune est hybride, miperceptive mi-linguistique. Talmy la décompose en quatre éléments. La dimension perceptive (et thématique) est constituée de deux éléments : la figure, c'est-à-dire 
l'actant dont la localisation est à déterminer et le fond l'actant servant à repérer la figure..$^{13}$ Le composant appelé Motive est un verbe générique profond (BE ou MOVE, selon que la localisation est statique ou dynamique). La nature de la relation spatiale de la figure et du fond est donnée par le composant dit Directional.

L'exemple ci-après illustre une dérivation. La figure profonde est RAIN ; ce « concept » se déplace et vient s'adjoindre au composant processif Motive pour former le verbe rain:

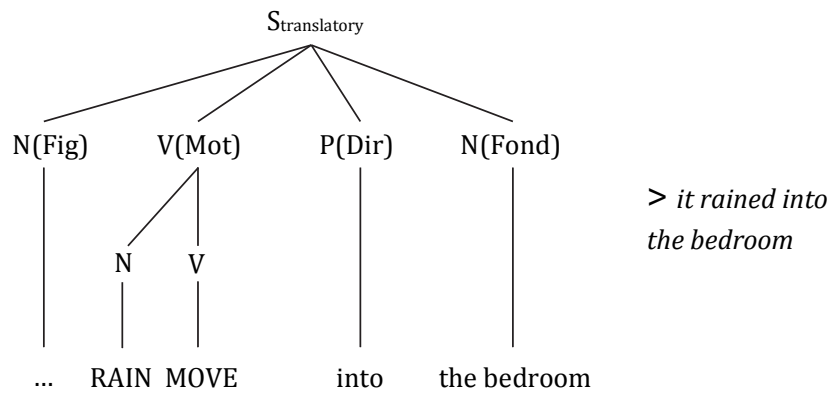

Un exemple d'adjonction dans une dérivation (Talmy,1972, p. 24).

Ce type d'adjonction est ce que Talmy appelle une conflation (une « fusion »). Il a d'évidentes affinités avec la transformation de montée du prédicat en sémantique générative. D'autres transformations permettent de rendre compte de fusions avec des composants sémantiques externes, par exemple la manière. Talmy postule ainsi que le verbe to float est formé par la fusion de MOVE avec un adverbe de manière externe afloat.

L'anglais et l'atusgewi lexicalisent de manière différente des informations souvent dissemblables. Le noyau du verbe polysynthétique atsugewi est typiquement formé de bases « classificatrices » qui qualifient la figure, le fond, le mode de déplacement, l'instrument du déplacement, mais ne spécifient pas la relation spatiale indépendamment. C'est cette diversité linguistique d'ordre sémantique qui semble intéresser Talmy au premier chef. Elle est parfois décrite, et le sera de plus en plus, en termes de relief attentionnel, et la lexicalisation envisagée comme une procédure de focalisation ou de « défocalisation » de tel ou tel composant sémantique.

13 Peut-être inspiré par Whorf : «Pour comparer les manières qu'ont différentes langues de "découper" différemment la même situation ou expérience, il est souhaitable de pouvoir analyser ou "découper" d'abord l'expérience indépendamment de toute langue particulière ou famille linguistique, de façon que ce découpage soit le même pour tous les observateurs. [...] Il y a une chose sur laquelle s'accorderont tous les observateurs du phénomène "garçon qui court" [...] c'est qu'on peut le diviser en parties — et qu'ils aboutiront tous à la même division. Ils diviseront tous le phénomène en (1) une figure ou silhouette plus ou moins en mouvement (le garçon) et (2) une sorte d'arrière-plan ou champ contre lequel ou dans lequel la figure est vue » (Whorf (1956) [1939], p. 162-163). Toutefois, le patron profond postulé par Talmy est quaternaire et son fond n'est donc pas exactement le fond gestaltiste. 
Comme Gruber, Talmy considère que les changements d'état sont conçus comme des mouvements : «Situations that involve state and change of state seem to be organized by the human mind in such a way that they can be specified by structures homologous with motion structures » (Talmy 1975, p. 234). Talmy étend ainsi la structure F-M-D-G à des situations non spatiales (extensions notées $\varphi-\rho-\delta-\gamma$ pour Figurid-Relator-Director-Groundid in Talmy 1972). Une situation « causative » est par exemple décomposée comme suit :

$\left[\text { the }_{\text {soot }} \text { fell }_{\mathrm{FM}} \text { into }_{\mathrm{D}} \text { the } \text { creek }_{\mathrm{G}}\right]_{\varphi}[\text { followed }]_{\rho}[\text { from }]_{\delta}[\text { the wind blowing on it }]_{\gamma}$ Cette structure profonde pourra donner, après dérivation (par ex. après fusion de fell et d'un FOLLOWED profond, ou fusion de la manière BLOW etc.) : the soot fell into the creek from the wind blowing on it, the soot blew into the creek from the wind (1972, p. 86-94).

Ce genre d'analyse menait à une décomposition fine des chaînes causales, décomposition qui avait pour conséquence que Talmy n'employait pas le prédicat CAUSE.

Les idées présentées par Talmy dans sa thèse sont directement à l'origine de la plupart des développements ultérieurs de sa théorie. L'évolution de son analyse de la causalité et du changement d'état pourrait illustrer l'absence de solution de continuité entre la forme de sémantique générative pratiquée dans cette thèse, et les développements postérieurs, qui voient Talmy s'engager dans une théorie cognitive de l'appréhension des relations causales (une sorte de physique naïve à l'usage des linguistes ; Talmy 1976, 1988b).

La structure profonde de 1972 a été conservée, non sans évoluer, et Talmy a réitéré depuis son hypothèse qu'il s'agit d'une structure « that is quite likely universal across languages, that at least in part corresponds to the structuring in other cognitive systems such as visual perception, and that may well be innately determined $\gg$ (Talmy 1996, p. 260 ; Fortis 2012).

La description sémantique des marqueurs spatiaux (verbes, préverbes, adpositions et particules), amorcée dans sa thèse, conduisit Talmy vers une description cognitive des modes de repérage employés par les langues, qui fut l'une des sources des études actuelles sur les cadres de référence (Talmy 1983 ; Levinson 1992). Finalement, son localisme, en particulier l'extension considérable que prit dans sa théorie l'asymétrie figure / fond, lui suggéra d'analyser les structures syntaxiques (par exemple l'asymétrie entre protase et apodose) comme des modes de mise en perspective de l'événement ayant un soubassement cognitif (Talmy 1988a).

En résumé, le virage cognitif pris par Talmy prolonge et infléchit les idées déjà présentes dans sa thèse. Élaborée dans un esprit proche de la sémantique générative, et en partie pour répondre au défi de comparer une langue polysynthétique à l'anglais, cette première étude a posé les fondements de la future sémantique cognitive de Talmy. Enfin, l'influence de Talmy sur les recherches consacrées à l'espace a été puissante. On peut soupçonner que Langacker s'est inspiré de lui pour généraliser l'emploi des notions de figure et fond dans sa grammaire cognitive (notions qui n'apparaissent chez lui qu'à partir de 1979 ; Fortis 2010c). 


\subsection{Catégories et prototypes}

La théorie du prototype, élaborée par la psychologue Eleanor Rosch dans les années 1970, a été importée en linguistique avant l'avènement de la linguistique cognitive, dans une étude de Lakoff (1973a). Adaptée, simplifiée et dégagée du formalisme que Lakoff lui avait adjoint (la théorie des ensembles flous), la théorie du prototype est par la suite devenue un des principaux outils de l'arsenal descriptif de la linguistique cognitive.

4.3.1. Catégories et prototype : la théorie de Rosch

La théorie a été construite progressivement, par agglomération successive de concepts et élargissement de son champ (Fortis 2010b). À l'origine, Rosch relayait une série de recherches sur les couleurs, menées notamment à Harvard par Eric Lenneberg et Roger Brown, et dont l'objectif était de tester expérimentalement ce qu'on avait convenu d'appeler l'hypothèse de Sapir-Whorf (et que Whorf, à la suite de Sapir, appelait le principe de relativité). Animées initialement d'un esprit relativiste, ces recherches s'étaient ensuite orientées vers une vision universaliste de la catégorisation des couleurs.

Dans ce contexte paraît en 1969 la célèbre étude de Berlin et Kay, une enquête linguistique sur la dénomination des couleurs, avec un échantillon d'une ampleur inégalée (elle portait sur 98 langues, mais les données étaient de première main pour vingt langues seulement). Rappelons brièvement les résultats de l'étude. Selon Berlin et Kay, le lexique des couleurs d'une langue est construit sur des «termes de base » (basic terms) dont la signification centrale correspond à des couleurs focales qui sont uiverselles, c'est-à-dire sont identiques pour les termes de base de langues différentes désignant la même région du spectre. Berlin et Kay identifient en outre plusieurs systèmes récurrents de termes de base, qu'ils interprètent comme autant de stades dans une évolution qui mène d'un système dual (réduit à blanc / noir) à un système pleinement différencié de 11 couleurs dénommables par des termes de base. Parce qu'elles paraissent être universelles, les couleurs focales suggèrent l'existence d'un déterminisme biologique (Berlin et Kay 1969, p. 109). Ce déterminisme recevra le renfort de Kay et MacDaniel (1978), qui proposeront une modélisation neurophysiologique d'une version modifiée de la théorie de Berlin et Kay (1969).

Critiquables du point de vue méthodologique (Hickerson 1971, Saunders 1992, Dubois \& Cance 2009 et ce numéro), mais chevauchant une vague universaliste (Saunders 1992), les résultats de Berlin et Kay apparurent à certains et à Rosch en particulier comme une remise en cause de la relativité linguistique.

Rosch profita d'une mission en Nouvelle-Guinée de son mari, l'anthropologue Karl Heider, pour tester in vivo les thèses de Berlin et Kay. Très opportunément, la langue sur laquelle elle travailla, le dani, n'avait « esssentiellement » que deux termes de couleurs (qu'elle assimila aux blanc et noir de Berlin et Kay). ${ }^{14}$ Elle montra que les Danis attribuaient à des régions du spectre les mêmes couleurs focales que des anglophones, en dépit de l'absence de termes de base pour ces

14 Le dani a en réalité plus de deux termes de couleurs, comme le signalent Rosch elle-même et Karl Heider dans son livre ethnographique, mais Rosch choisit les sujets qui utilisaient essentiellement ces deux termes (Heider 1970, Rosch 1972b, Fortis 2010b). 
régions dans leur langue (Rosch 1972a). Cette observation la conduisit vers les mêmes conclusions anti-relativistes que Berlin et Kay et, comme eux, vers l'hypothèse d'un déterminisme cognitif.

Rosch élargit ensuite sa recherche aux formes visuelles et employa, pour désigner à la fois les couleurs focales et les bonnes formes (au sens gestaltiste), le terme de «prototype », qui avait été employé en psychologie pour désigner un schéma générateur d'une série de stimuli (Rosch 1973, Fortis 2010b sur ce lien entre schéma et prototype). Élargissant encore son champ d'investigation (Rosch 1973b), elle se tourna rapidement vers ce qu'elle appela les catégories " sémantiques », c'est-à-dire des catégories envisagées d'un point de vue taxinomique, par exemple la catégorie « chien » considérée comme dénomination de l'ensemble des races et des individus canins. C'est une étude préliminaire sur ces catégories « sémantiques » (Rosch 1971) qui suggéra à Lakoff d'importer la théorie du prototype en linguistique.

Les catégories «sémantiques » sont différentes des couleurs et des formes : elles n'ont a priori pas de fondement neurophysiologique et Rosch suppose qu'elles doivent être acquises par apprentissage à partir des membres de la catégorie et d'un processus de traitement de l'information. D'autre part, leur solidarité interne pose problème : qu'est-ce qui unit au sein d'une même catégorie « sémantique » OISEAU des exemplaires aussi différents qu'un pingouin et un moineau ? Enfin, les catégories sémantiques sont à différents d'abstraction, ANIMAL étant par exemple supérieur à CHIEN dans la taxinomie.

Au terme d'emprunts théoriques successifs (Fortis 2010b pour plus de détails), le traitement de ces questions conduisit Rosch aux positions suivantes : les catégories « sémantiques » sont organisées comme les catégories «naturelles », autour de prototypes (tous les membres ne sont pas également typiques de la catégorie, ils sont associés par une ressemblance de famille) ; la catégorisation est probabiliste, ce qui signifie que les indices permettant d'affecter un item à une catégorie ont une force probatoire (ou cue validity, validité d'indice) plus ou moins élevée ; enfin, il existe un niveau privilégié de catégorisation, le niveau de base, où la discontinuité morphologique (perceptive et motrice) du référent par rapport au niveau supérieur est maximale, et où les validités d'indice cumulées sont maximales ${ }^{15}$; les hiérarchies taxinomiques sont donc, pour ainsi dire, formées «naturellement » (Rosch 1976, 1977, 1978 ; Dubois \& RescheRigon 1995 et Dubois \& Cance, ce numéro, pour une critique de cette notion de naturalité).

4.3.2. La théorie du prototype en linguistique (1) : grammaire floue et ensembles flous

Comme je l'ai mentionné, le premier emprunt de la théorie du prototype est dû à Lakoff (1973a), à une époque où il développe avec John Ross un modèle grammatical nouveau, la « grammaire floue » (fuzzy grammar). Dans ce modèle, l'acceptabilité d'une construction dépend d'une hiérarchie de contraintes syntaxiques et lexicales

15 Le niveau de base a été inspiré à Rosch par Berlin (cf. Berlin 1973), qui mettait en évidence, dans plusieurs cultures, la saillance d'un niveau taxinomique particulier, appelé folk genus. Chez Rosch, le niveau de base s'est d'ailleurs appelé d'abord niveau générique (Rosch 1975). Cependant, folk genus et niveau de base ne concordent pas complètement (Fortis 2010b). 
(Lakoff 1973b). Par exemple, la faculté pour un adverbe de se préposer dépend d'une hiérarchie de mobilité des adverbes et d'une hiérarchie de fermeture des îlots :

(5) a. Tomorrow it's likely that Sam will leave town.

a'. ? In Berkeley it's likely that there will be a riot. [l'adv. de temps est moins mobile que l'adv. de lieu]

b. Tomorrow I think that John will leave.

b'. * Tomorrow I'm surprised that John will be in his office. [pour un même adverbe, I'm surprised that est un îlot plus fermé que I think that]

Cette notion de flou pouvait s'appliquer à la sémantique lexicale, en partant de l'idée que l'appartenance d'un item à une catégorie (par exemple de la baleine à la catégorie des mammifères) variait de 0 à 1 , c'est-à-dire définissait un ensemble flou (au sens de Zadeh 1965). Les degrés d'appartenance à une catégorie variant, le problème de la solidarité interne de la catégorie pouvait être appréhendé au moyen de la théorie de Rosch. Les enclosures ${ }^{16}$ (hedges) fournissaient en outre un test linguistique permettant d'estimer non seulement le degré d'appartenance à une catégorie, mais le type de propriété (définitoire ou non) conditionnant la catégorisation. Pour les noms, Lakoff opposait par exemple l'enclosure strictly speaking (strictly speaking, a whale is a mammal), appropriée pour des instances ayant un haut degré d'appartenance à une catégorie, à l'enclosure regular, applicable à des instances ayant un degré d'appartenance nulle à une catégorie, comme par exemple dans Harry is a regular fish (Harry n'appartient pas à la catégorie poisson). D'un autre côté, Lakoff assignait à ces enclosures la capacité d'isoler, de « catalyser » des types d'attributs différents (définitoires, propres à la catégorie pour strictly speaking, ou bien « connotatifs », métaphoriques, non critériaux, pour regular).

4.3.3. La théorie du prototype en linguistique (2) : sémantique lexicale La théorie du protoype a été adoptée en sémantique lexicale afin de satisfaire deux objectifs principaux.

Le premier objectif, qui prolonge celui de Lakoff (1973a), a été de rendre compte de la catégorisation «floue ». Il s'agit ici des cas où un objet ou une situation satisfont à des degrés divers la définition d'un prototype, et où des traits « connotatifs » et non définitoires interviennent dans la catégorisation. Cet objectif inspire l'étude de Coleman et Kay (1981) sur le verbe lie «mentir » et ses conditions d'applications. Sans l'appareil formel de Lakoff (1973a), Coleman et Kay poursuivent au fond les mêmes buts que lui.

Le second objectif a été de rendre compte de la polysémie, sujet qui était marginal dans les années 1970 (si l'on excepte la thèse de Nunberg 1978). L'idée directrice était que les diverses acceptions d'un lexème, comme les membres d'une catégorie roschienne, sont liées entre elles par une ressemblance de famille et organisées autour d'un prototype central.

Une des premières études appliquant la théorie du prototype au traitement de la polysémie fut celle de Brugman (1981), qui portait sur la préposition et particule over. Cette étude avait plusieurs caractères remarquables, que nombre d'études

16 La traduction de hedge par enclosure a été proposée par Kleiber et Riegel (1978). 
postérieures partagent avec elle : elle était sémasiologique, ne faisait pas appel à un corpus défini et étendu, et défendait, dans un esprit localiste, la primauté des sens spatiaux. En outre, la théorie expliquait l'extension sémantique par la métaphorisation, et défendait une analyse des sens prototypiques en termes de gestalts, illustrées par des diagrammes. L'étude de Brugman fut revue et systématisée dans le best seller que Lakoff consacra à la catégorisation (1987, près de 100 000 exemplaires, cf. Peeters 2001). Elle déclencha une vague de travaux qui en contestèrent l'analyse de détail sans remettre en cause son approche (Fortis 2009).

En passant de Rosch à la sémantique cognitive, la théorie du prototype a perdu une notion, celle de la validité d'indice, qui, rappelons-le, enregistrait la valeur contrastive d'un trait de la catégorie (et posait d'ailleurs problème à Rosch) ${ }^{17}$. Ainsi, la sémantique cognitive s'est peu intéressée aux lexèmes comme membres de systèmes. En outre, une fois l'emprunt effectué, elle s'est désintéressée de l'évolution de la théorie de la catégorisation en psychologie. Plus surprenant peutêtre, la sémantique cognitive n'a guère collaboré avec la psychologie ni soumis à l'épreuve expérimentale les analyses obtenues avec l'intuition (ce qui justifie le scepticisme de Sandra \& Rice 1995). En réalité, l'importation de la théorie du prototype a permis de traiter à peu de frais la polysémie, c'est-à-dire sans méthode de validation exigeante.

Il convient de remarquer que ces recherches ignorent largement les prédécesseurs (que Geeraerts a depuis rappelés à notre souvenir ; cf. Geeraerts 1988, 2010). Même l'étude sophistiquée de Labov (1973), qui remonte à des travaux antérieurs à Rosch, n'est mentionnée que de façon marginale par les linguistes cognitivistes (un codicille chez Lakoff 1987).

4.3.4. La théorie du prototype en linguistique (3): schéma et formation analogique Au-delà de la sémantique lexicale, une des motivations de l'importation de la théorie du prototype est qu'elle a permis de récupérer la thématique de la formation analogique (cf. Legallois \& François 2011). Dès 1980, Zager proposait, dans sa thèse de doctorat, de substituer des schémas prototypiques à la notion de règle mettant en relation une forme de base et une forme dérivée. Bybee, qui a dirigé la thèse de Zager, et Slobin (Bybee \& Slobin 1982 ; voir aussi Bybee \& Moder 1983) adoptent l'idée et l'appliquent aux passés des verbes irréguliers anglais, considérant que les passés des verbes irréguliers peuvent être classés en différentes sous-familles dont chacune est une catégorie à la Rosch. Ils identifient par exemple une classe de verbes formée sur le schéma [...in ... > ... æy...], et à laquelle appartiennent les formes sang, sprang, rang. L'attraction exercée par le prototype (le schéma [... $\left.æ \eta(\mathrm{k})]_{\text {verb/ past }}\right]$ ) rend compte à la fois des formes dialectales (comme bring $>$ brang) et des « erreurs » produites par les locuteurs (string > strang).

Cette approche permet de connecter la linguistique cognitive avec les recherches sur la variation et la diachronie. En outre, du fait que les schémas sont considérés comme des formations produites par un processus d'extraction de régularités, les thématique de l'usage et de la généralité par abstraction deviennent de plus en

17 À ma connaissance, Nunberg (1978) est le seul linguiste à employer la validité d'indice dans sa sémantique. Cette notion remonte à la théorie probabiliste de la perception élaborée par le psychologue d'origine autrichienne Egon Brunswik (élève de Bühler et membre du Cercle de Vienne), qui émigra aux États-Unis en 1937 (Fortis 2010b). 
plus importantes ${ }^{18}$. C'est ainsi que Langacker (1988) rend compte des catégories et des structures abstraites (par ex. la structure en constituants) par un processus de « schématisation », c'est-à-dire d'abstraction empiriste.

Enfin, les notions de schéma et de prototype vont être employées par Goldberg (1995), élève de Fillmore, pour rendre compte des variantes de la construction ditransitive en anglais (du type give $X Y$ ) et dépasser certaines apories soulevées auparavant par Green (1974), qui travaillait sur cette construction dans le cadre de la sémantique générative mais manquait d'une théorie de la polysémie.

Ainsi, schéma, prototype et construction concourent progressivement à définir une approche cohérente, «empiriste », où les structures linguistiques sont formées par abstraction à partir de données fréquentes ou saillantes (usage-based grammar dit Langacker). La langue tend ainsi à être envisagée comme un inventaire où coexistent le particulier, l'idiomatique, le mémorisé, ou le formulaire, et les généralisations ou procédures de dérivation et d'inflexion (les « règles »). Cette vision contraste avec le générativisme, qui tend à opposer mémorisé, formulaire et idiosyncratique à dérivable et général. Elle a reçu le double soutien de la théorie des métaphores et du localisme, qui, comme la théorie du prototype, tendent à défendre une vision empiriste de la signification.

\subsection{La notion de cadre (frame)}

La notion de cadre a eu une grande influence, dont je dirai quelques mots, et a donné lieu à un projet innovant de dictionnaire. Introduite par Fillmore (1968), cette notion a ensuite fusionné avec des concepts apparentés, comme nous allons le voir.

4.4.1. Le cadre casuel

Fillmore emploie d'abord cadre pour décrire la structure actancielle ou « casuelle » (case frame), dans une théorie où les énoncés de surface sont engendrés à partir de telles structures. Entre autres motivations, la primauté des rôles actanciels et des cas profonds qui les expriment se justifie par le souci de rendre compte de la variété typologique, c'est-à-dire de formuler une théorie plus générale et moins « anglo-centrée » que la grammaire générative.

Sa date d'émergence situe la théorie fillmorienne des « cas » dans le mouvement plus global auquel appartient la sémantique générative, c'est-à-dire un mouvement qui fait des structures sémantiques le noyau d'où proviennent les formes de surface. Mais, à la différence des sémanticiens générativistes, les influences que Fillmore revendique sont multiples, et parmi elles, celle de Tesnière semble se distinguer. Il faut ainsi souligner que la formation de Fillmore n'est pas imprégné de générativisme au degré où l'a été celle d'un Lakoff ou d'un Langacker. Sa culture est assez éclectique (l'article de 1968 cite Kuryłowicz, Van Ginneken, Lévy-Bruhl...).

Chez Fillmore, l'assignation d'un cas à un argument est une affaire assez complexe, où intervient d'emblée le sémantisme particulier du verbe. Par exemple,

18 La situation actuelle des approches fondées sur l'usage est très bien exposée par Legallois \& François (2011). 
le verbe break impose des restrictions sur les objets qui peuvent être patients, et ces restrictions sont distinctes de celles qui sont imposées par le verbe hit. Ces différences, qui se reflètent dans le comportement syntaxique (voir (6) et (7)), peuvent être partiellement exprimées en disant que break a dans sa structure actancielle un patient (object) là où hit a un lieu (place).

(6) *I broke him on the leg. [« montée du possesseur»; à rapprocher de I broke his leg]

(7) I hit him on the leg. [« montée du possesseur »; à rapprocher de I hit his leg]

Le besoin de distinguer finement les rôles actanciels constitue une première étape vers l'enrichissement du cadre (frame).

\subsubsection{Champs et scènes}

$\mathrm{Au}$ stade suivant de son évolution, Fillmore se tourne vers un niveau de description qui est intermédiaire entre les cas, dont le contenu est très général, et la sémantique du verbe, dont le contenu est très spécifique. Ce niveau peut être décrit comme correspondant à un champ sémantique (par ex. celui des verbes de jugement, comme accuser ou critiquer; Fillmore 1970), ou un schéma (schema ; Fillmore 1977b), entendu comme structure conceptuelle (préconditions, actants, actions...) qui articule une scène de l'expérience, et dont le cadre est l'expression linguistique. L'exemple canonique d'une scène est celle de l'échange commercial, dont le schéma sert de contexte d'interprétation aux verbes buy et sell.

Ce que les verbes qui appartiennent à un champ sémantique ou réfèrent à une scène partagent est une structure de connaissances constituée d'actants qui entrent dans un scénario type. Ce que les verbes eux-mêmes expriment, c'est une manière de configurer cette structure de connaissances. Par exemple, la structure de connaissances associée aux verbes accuser et critiquer pose trois actants centraux, l'accusateur, l'accusé et la situation, auxquels s'ajoute éventuellement un destinataire de l'acte de communication ; ces actants participent à un scénario dans lequel un accusateur émet un jugement moral sur la responsabilité de l'accusé dans la situation, et communique éventuellement ce jugement à un destinataire (Fillmore 1970). Accuser diffère de critiquer en ce qu'accuser asserte la responsabilité de l'accusé et présuppose que la situation est négative, alors que critiquer asserte que la situation est négative et présuppose que l'accusé est responsable.

Toute cette discussion des structures de connaissance se poursuit sur le fond d'une réflexion sur ce que devrait être une approche scientifique du lexique (Fillmore 1969). Comme une telle approche exige de prendre en compte la construction syntaxique des lexèmes, Fillmore esquisse en ces années la future grammaire de construction, non sans se souvenir, d'ailleurs, de ses débuts distributionnalistes ${ }^{19}$ (Fillmore 1982).

19 Lorsqu'il était étudiant à l'université du Michigan, où l'influence de Charles Fries était forte, Fillmore a participé à un projet d'analyse distributionnelle. Il a collaboré ensuite à un projet de classification des verbes anglais en fonction de leurs types constructionnels. On sait que Fries était un défenseur du pattern matching en apprentissage d'une langue seconde, et qu'il s'était associé à Pike, le pape de la tagmémique. La notion de construction s'inscrit donc aussi dans le distributionnalisme. 
4.4.3. La scène comme cadre d'interprétation

Une des préoccupations constantes de Fillmore a été de rendre compte de la projection des rôles actanciels sur les relations grammaticales, et donc de formuler des règles permettant de rendre compte du fait, par exemple, que le sujet est tantôt l'agent, le patient ou l'instrument.

Au fil des années, les règles simples du début sont d'une complexité croissante, et qui reflète l'évolution de la linguistique. Les années 1970 voient se développer les travaux de linguistique fonctionnelle, la grammaire relationnelle, les recherches sur la topicalité et le focus, sur la typologie, dont Fillmore reprend des éléments. La conséquence est la multiplication des facteurs susceptibles d'influencer la projection des rôles sur les relations grammaticales : les relations de sujet et d'objet direct, explique-t-il, sont associées non seulement aux agents et aux patients, mais au rôle d'expérienceur, à la « saillance » d'un objet, au degré d'objectification, ou à l'inverse d'empathie, avec une entité animée, à l'affectation plus ou moins totale d'un actant lors du procès. Encore reste-t-il des idiosyncrasies, des « exceptions » difficilement explicables (Fillmore 1977b).

D'autre part, nous l'avons vu, Fillmore caractérise aussi les actants en termes très spécifiques, relatifs à un type de situation (comme «accusateur » ou « accusé » etc.). Ainsi, les cas généraux expliquent de moins en moins le problème de la projection des rôles actanciels, et n'entrent plus dans la description des scènes, de sorte qu'ils n'expriment plus que des tendances, ou des généralisations tendancielles sur des scènes : l'actant actif tend à primer sur l'actant inactif, l'expérienceur tend à primer sur les autres actants etc. Ces tendances doivent en outre être ordonnées (l'agent prime sur l'expérienceur, par exemple). Les dérivations de type générativiste sont une victime collatérale de ce changement, puisqu'elles sont fondées sur la constituance (non sur les fonctions grammaticales) et sur une liste limitée de cas.

Le résultat de cette évolution semble être une attention de plus en plus grande à la structure sémantique des scènes, et aux spécificités sémantiques des lexèmes qui quadrillent un type de scène (par exemple, pour la scène de l'échange financier : frais, honoraires, pension, pourboire, rançon, redevance, salaire, etc. ; Fillmore 1977b).

Enfin, la notion de scène-cadre fait jonction avec celle de prototype. Dans l'esprit de Fillmore, cadre et typicalité semblent fortement liés : le cadre nécessaire à la compréhension de bachelor est un stéréotype, non une définition stricte (Fillmore 1975), ce qui explique aussi la difficulté de catégoriser les cas déviants, trop éloignés des stéréotypes. Ce lien entre cadre et typicalité, qu'on retrouvera chez Lakoff (1987), tient aussi à la primauté de l'expérience du corps propre et des scènes concrètes dans la sémantique et son acquisition (Fillmore 1975, 1977b).

4.4.4. Le cadre au sens étendu

La notion de cadre finit par prendre une extension très large. Elle correspond à la notion de champ sémantique, de situation ou de scénario-type, d'échelle de valeurs dans un domaine (par ex. stingy péjoratif et thrifty mélioratif), de mode de conceptualisation (par ex. perceptif ou procédural pour angle), de « point de vue » (la mer pour shore, la terre pour coast), de théorie : «By the term frame », explique Fillmore, «I have in mind any system of concepts related in such a way that to 
understand any one of them you have to understand the whole structure in which it fits » (Fillmore 1982, p. 111). Application (parmi d'autres) de cette extension, dans le contexte générativiste, le cadre permettait aussi de traiter l'épineuse question des noms composés, impossibles à engendrer et à traiter sémantiquement par des mécanismes réguliers, tant la forme, une simple juxtaposition, sous-détermine la nature du lien sémantique (voir Downing 1977, dont Fillmore 1982 revendique l'importance). En l'occurrence, le cadre poursuit le même objectif que la gestalt de Lakoff (1977).

Toujours soucieux de décrire conjointement la scène, le cadre linguistique qui structure une scène et les relations grammaticales, Fillmore s'est par la suite engagé dans une analyse sur corpus qui conjugue ces trois niveaux et qui a débouché sur le projet FrameNet, un dictionnaire électronique fondé sur les cadres (Ruppenhofer et al. 2006).

4.4.5. Cadres: sociologie et intelligence artificielle

Dans les années 1970, d'autres auteurs emploient le terme de frame. En dehors de la linguistique, la notion a de multiples origines (Nerlich \& Clarke 2000). Limitonsnous ici aux deux sources les plus importantes, la sociologie et l'intelligence artificielle.

Le sociologue Erving Goffman (1974) emploie frame pour désigner un «principe d'organisation », de sélection, et de mise en rapport d'éléments de l'expérience qui permet d'interpréter « ce qui se passe » (« ... while one thing may momentarily appear to be what is really going on, in fact what is happening is plainly a joke, or a dream, or an accident, or a mistake, or a misunderstanding, or a deception, or a theatrical performance, and so forth »; Goffman 1974, p. 10). C'est principalement ce sens de frame que Lakoff, qui connaît Goffman depuis ses débuts, semble avoir à l'esprit quand il discute des idéologies contenues dans des systèmes métaphoriques et de la propagande politique (Lakoff 1972, Lakoff \& The Rockridge Inst. 2006, ch. 3).

En intelligence artificielle, plusieurs chercheurs s'efforcent à cette époque de rendre compte de la compréhension de textes (Van Dijk 1977), ou de l'organisation de séquences d'action et des connaissances sur l'action (Norman \& Rumelhart 1975, Schank \& Abelson 1977) en postulant des systèmes de concepts qui sont activés lorsqu'un élément du système est appelé. Les scripts de Schank et Abelson, par exemple, sont des scénarios types formulés au moyen de primitives conceptuelles. Quant à Minsky (1975), il introduit le concept de cadre dans le but, encore programmatique, de modéliser le raisonnement humain dans des contextes stéréotypés.

4.4.6. Influence de la notion de cadre

L'influence de la notion de cadre est sensible chez Lakoff et Langacker. Lakoff a par la suite tenté de subsumer les notions de stéréotype, de cadre et de système métaphorique sous celle de modèle cognitif idéalisé (Idealized Cognitive Model) mais cette tentative n'a guère eu de postérité (Lakoff 1982, 1987). La notion de cadre a vraisemblablement inspiré la sémantique de Langacker qui, dans sa grammaire cognitive, identifie la signification à une relation entre un composant sémantique et le domaine qui permet de l'interpréter. Le domaine en question évoque irrésistiblement le cadre fillmorien. 


\section{CONCLUSION}

Aux doutes qui ont pu s'exprimer sur la possibilité même d'identifier une linguistique cognitive (Lazard 2007), ce qui précède permet de fournir une réponse, sans doute encore à développer (voir aussi Fuchs 2009). Les approches théoriques que nous avons décrites sont issues d'un même environnement intellectuel, marqué par la sémantique générative et ont évolué vers une «psychologisation » de la linguistique vers la même époque. Elles se sont influencées l'une l'autre, s'empruntant des outils descriptifs (figure et fond, prototype, schéma, cadre, métaphore, pour nous limiter aux notions abordées ici), ou partageant une orientation qu'on pourrait qualifier d'empiriste, c'est-à-dire privilégiant l'expérience du monde physique dans l'appréhension des abstracta. Conscient de son unité, ce mouvement s'est doté d'une forme institutionnelle à la fin des années 1980, par la création d'une association internationale de linguistique cognitive et la parution de la revue Cognitive Linguistics (respectivement en 1989 et 1990 ; voir l'introduction à ce numéro).

Le tournant cognitif correspond à ce moment où les linguistes dont nous avons parlé ici abandonnent la génération de structures en constituants au moyen de règles de dérivation ou, s'ils continuent à s'en soucier, délaissent définitivement les opérations ou marqueurs formels, le formalisme logique, la recherche de procédures de génération qui seraient sans portée sémantique, sans fonction cognitive ou communicative.

Plus positivement, le tournant cognitif est ce moment où des théories convergent vers les positions suivantes :

1) le problème central de la linguistique est la sémiose (la mise en forme linguistique de la signification). Cette orientation est déjà présente en sémantique générative.

2) Les structures linguistiques lexicalisent des représentations des objets et des événements (ce qui implique que la linguistique s'intéresse à des modèles conceptuels du monde, par ex. de la causalité physique, et s'articule ainsi avec une physique naïve). Il s'agit en quelque sorte d'approfondir les représentations sémantiques (par ex. le prédicat CAUSE) postulées en sémantique générative, et d'ouvrir la sémantique à l'expérience du monde (systèmes de croyance, gestalts, modèles, cadres et scènes).

3) Les notions grammaticales et structures fondamentales sont souvent exprimées en termes localistes ou relevant du perceptif, du proprioceptif, de la motricité et en général des interactions homme-environnement (embodiment, thèmes gestaltistes, empiristes, pragmatistes). Des théories d'inspiration localiste (chez Gruber et Talmy) sont déjà présentes dans la galaxie générative, mais le motif empiriste ou « expérientialiste » prend une ampleur nouvelle.

Enfin, et ce sont des points sur lesquels nous n'avons pu insister suffisamment :

4) la linguistique cognitive fait appel à des facultés psychologiques (imagerie, schématisme, abstraction / catégorisation, attention, mémoire) ; elle s'oppose ainsi à l'autonomie de la syntaxe.

5) La linguistique cognitive est inductiviste : elle est opposée au nativisme, revalorise l'idiomaticité, l'usage, l'analogie, réintroduit la notion de 
construction. À une époque récente, cette orientation a favorisé le regain de la linguistique de corpus.

6) La linguistique cognitive est non- voire anti-formaliste, et ne recherche pas l'élégance computationelle (la réduction ou la simplification des règles, la productivité maximale etc.).

Ces positions justifient qu'on regarde la linguistique cognitive comme une forme d'anti-générativisme. Cependant, la prise en compte des conditions historiques d'émergence de la linguistique cognitive doit conduire à nuancer ce diagnostic. J'espère que les éléments fournis dans cet article permettent de le montrer.

\section{RÉFÉRENCES}

Anderson, John M. (1971). The grammar of case: Towards a localistic theory, London, Cambridge University Press.

Ariel, Mira (2010). Defining pragmatics, Cambridge, Cambridge University Press.

Bach, Emmon (1968). « Nouns and Noun Phrases », Bach, Emmon \& Harms, Robert T. (eds), Universals in Linguistic Theory, New York, Holt, Rinehart and Winston, 90-122.

Bamberg, Michael (1983). « Rezension von George Lakoff / Mark Johnson, Metaphors we live by », Zeitschrift für Sprachwissenschaft 2(1), 144-148.

Berlin, Brent, Breedlove, Dennis E. \& Raven, Peter H. (1973). « General principles of classification and nomenclature in folk biology », American Anthropologist 75, 214242.

Berlin, Brent \& Kay, Paul (1969). Basic color terms: their universality and evolution, Berkeley, University of California Press.

Black, Max (1955). « Metaphor », Proceedings of the Aristotelian Society 55, 273-294.

Black, Max. (1962). Models and Metaphors: Studies in Language and Philosophy, Ithaca (NY), Cornell University Press.

Black, Max (1979). «More about metaphor», Ortony, Andrew (ed.), Metaphor and thought, Cambridge, Cambridge University Press, 19-43.

Bolinger, Dwight (1961). « Syntactic blends and other matters », Language 37(3), 366-381.

Bolinger Dwight (1976). « Meaning memory », Forum Linguisticum 1(1), 1-14.

Bolinger, Dwight (1977). Meaning and form, London \& New York, Longman.

Borkin, Ann (1972). « Coereference and beheaded NP's », Papers in Linguistics 5, 28-45.

Brugman, Claudia (1981) Story of Over, M.A. Thesis, University of California, Berkeley.

Burkhardt, Armin \& Nerlich, Brigitte (2010). «Introduction », Burkhardt, Armin \& Nerlich, Brigitte (ed.), Tropical truths, Berlin/New York, De Gruyter, 1-20.

Bybee, Joan L. \& Moder, Carol Lynn (1983). « Morphological Classes as Natural Categories », Language 59(2), 251-270.

Bybee, Joan L. \& Slobin Dan I. (1982). « Rules and Schemas in the Development and Use of the English past Tense », Language 58(2), 265-289.

Chafe, Wallace L. (1962). « Phonetics, semantics, and language », Language 38(4), 335-344.

Chafe, Wallace L. (1965). «Meaning in language », American Anthropologist 67(5), 23-36.

Chafe, Wallace L. (1967). « Language as symbolization », Language 43(1), 57-91.

Chafe, Wallace L. (1970a). Meaning and the structure of language, Chicago, University of 
Chicago Press.

Chafe, Wallace L. (1970b). A semantically based sketch of Onondaga, Baltimore, Indiana University Publications in Anthropology and Linguistics.

Chafe, Wallace L. (1971). « Directionality and paraphrase », Language 47(1), 1-26.

Chafe, Wallace L. (1973). «Language and Memory », Language 49(2), 261-281.

Chafe, Wallace L. (1974). «Interview », Parret, Herman (dir.), Discussing language, The Hague, Mouton, 1-25.

Chafe, Wallace L. (1979). « The Flow of Thought and the Flow of Language », Givón, Talmy (ed.), Discourse and Syntax, New York, Academic Press, 159-181.

Chafe, Wallace L. (2002). « Searching for meaning in language: a memoir », Historiographia Linguistica 29, 245-261.

Chomsky, N., (1969) [1957]. Structures syntaxiques, Paris, Le Seuil [tr. fr. de Syntactic structures, Paris/The Hague, Mouton].

Clark, Herbert H. (1973). «Space, time, semantics and the child », Moore, Timothy E. (ed.), Cognitive development and the acquisition of language, New York, Academic Press, 27-63.

Col, Gilles (2010) «Correspondance et mixage d'espaces mentaux dans la construction dynamique du sens », François, Jacques (dir.), Grandes voies et chemins de traverse de la sémantique cognitive, Mémoires de la Société de Linguistique de Paris 18, 53-73.

Coleman, Linda \& Kay, Paul (1981). « Prototype semantics: the English word lie », Language 57(1), 26-44.

Danesi, Marcel (2001). Lingua, metafora, concetto. Vico e la linguistica cognitiva, Bari, Edizioni dal Sud.

Davidson, Donald (1984) [1978]. «What metaphors mean », Inquiries into Truth and Interpretation, Oxford, Oxford University Press, 245-264.

Downing, Pamela (1977). « On the creation and use of English compound nouns », Language 53(4), 810-842.

Dubois, Danièle \& Cance, Caroline (2009). « Mettre un terme aux couleurs de base », Dubois, Danièle (dir.), Le sentir et le dire, Paris, L'Harmattan, 75-104.

Dubois, Danièle \& Resche-Rigon, Philippe (1995). « De la "naturalité" des catégories sémantiques : des catégories "d'objets naturels" aux catégories lexicales ", Intellectica 20, 217-45.

Fauconnier, Gilles (1978). «Espaces référentiels », Sull’Anafora, Academia della Cruzca, Florence, 129-146.

Fauconnier, Gilles (1984). Espaces mentaux. Aspects de la construction du sens dans les langues naturelles, Paris, Minuit.

Fillmore, Charles J. (1968). « The case for case ». Bach, Emmon \& Harms, Robert T. (eds), Universals in Linguistic Theory, New York, Holt, Rinehart \& Winston, 1-88.

Fillmore, Charles J., (2003) [1969]. « Types of lexical information », Fillmore, Charles J., Form and meaning in language, vol. 1, Stanford, CSLI Publications, 141-174 [repris de Kiefer, F., (ed.), Studies in Syntax and Semantics, Reidel, Dordrecht, 109-137].

Fillmore, Charles J. (1970). «Verbes de jugement. Essai de description sémantique », Langages 17(5), 56-72.

Fillmore, Charles J. (1975). «An alternative to checklist theories of meaning », Papers from the First Annual Meeting of the Berkeley Linguistics Society, 123-132.

Fillmore, Charles J. (2003) (1977a). « The case for case reopened », Fillmore, Charles J., Form and meaning in language, vol. 1, Stanford, CSLI Publications, 175-200 [repris de Syntax and Semantics: Grammatical Relations 8(1), 59-81]. 
Fillmore, Charles J. (2003) [1977b] « Topics in lexical semantics », Fillmore, Charles J., Form and meaning in language, vol. 1, Stanford, CSLI Publications, 201-260 [repris de Cole, Roger W. (ed.), 1977, Current issues in linguistic theory, Indiana University Press, 76-138].

Fillmore, Charles J., (1982). « Frame semantics», The Linguistic Society of Korea (eds), Linguistics in the Morning Calm, Seoul, Hanshin, 111-137.

Fillmore, Charles J., Kay, Paul \& O'Connor, Mary Catherine, (1988). « Regularity and Idiomaticity in Grammatical Constructions: The Case of Let Alone », Language 64(3), 501-538.

Fodor, Janet Dean (1977). Semantics: Theories of Meaning in Generative Grammar, Crowell, New York.

Fortis, Jean-Michel (2009). «Le problème de la polysémie des adpositions spatiales », François, Jacques, Gilbert, Eric, Guimier, Claude \& Krause, Maxi (dir.), Autour de la préposition, Bibliothèque de Syntaxe et de Sémantique, Presses Universitaires de Caen, 183-193.

Fortis, Jean-Michel (2010a). «La linguistique cognitive, une trentenaire de vieille souche », François, Jacques (dir.), Grandes voies et chemins de traverse de la sémantique cognitive, Mémoires de la Société de Linguistique de Paris 18, 11-51.

Fortis, Jean-Michel (2010b). « De l'hypothèse de Sapir-Whorf au prototype : sources et genèse de la théorie d'Eleanor Rosch », CORELA, 8(2). [En ligne] http://corela.edel. univ-poitiers.fr/index.php?id=1243.

Fortis, Jean-Michel (2010c). «De la grammaire générative à la Grammaire Cognitive : Origines et formation de la théorie de Ronald Langacker », Histoire Épistémologie Langage 32(2), 109-149.

Fortis, Jean-Michel (2012). « Chafe et Talmy : Deux approches de la linéarisation à l'époque de la sémantique générative », Beiträge zur Geschichte der Sprachwissenschaft 22, 171-196.

Fortis, Jean-Michel (à paraître). « Localisme et théorie des cas ».

Fuchs, Catherine (2009). « La linguistique cognitive existe-t-elle ? », Quaderns de Filologia 14, 115-133.

Geeraerts, Dirk (1988). « Cognitive Grammar and the History of Lexical Semantics », Rudzka-Ostyn, Brygida (ed.), Topics in Cognitive Linguistics, Amsterdam-Philadelphia, John Benjamins, 647-677 [tr. fr. 1991, « La grammaire cognitive et l'histoire de la sémantique lexicale », Communications 53, 17-50].

Geeraerts, Dirk (2010). Theories of lexical semantics, Oxford, Oxford University Press.

Gerber, Gustav (1871-74). Die Sprache als Kunst, Vols. I/II, Bromberg, Mittler'sche Buchhandlung (2nd ed., 1885, Berlin, Gaertners Verlagsbuchhandlung. Reprint of the 2nd ed., 1961. Hildesheim, Georg Olms).

Goffman, Erving (1974). Frame analysis, Cambridge, Harvard University Press.

Goldberg, Adele (1995). Constructions: A Construction Grammar Approach to Argument Structure, Chicago, The University of Chicago Press.

Green, Georgia M. (1974). Semantics and syntactic regularity, Bloomington, Indiana University Press.

Gruber, Jeffrey (1965). Studies in lexical semantics, MIT Working Papers in Linguistics.

Harris, Randy Allen, (1993). The Linguistic Wars, Oxford, Oxford University Press.

Harris, Zellig, (1957). «Co-occurrence and transformation in linguistic structure », Language 33(3), 283-340.

Heider, Karl G. (1970). The Dugum Dani: A Papuan Culture in the Highlands of West New Guinea, Chicago, Aldine Publishing. 
Heine, Bernd, Claudi, Ulrike \& Hünnemeyer, Friederike (1991). Grammaticalization: A conceptual framework, Chicago, University of Chicago Press.

Herskovits, Annette (1986). Language and spatial cognition: an interdisciplinary study, Cambridge, Cambridge University Press.

Hickerson, Nancy P. (1971). « Review of Basic Color Terms: Their Universality and Evolution by Brent Berlin and Paul Kay », International Journal of American Linguistics 37(4), 257-270.

Huck, Geoffrey J. \& Goldsmith, John A. (1995). Ideology and Linguistic Theory: Noam Chomsky and the Deep Structure Debate, London, Routledge.

Hjelmslev, Louis (1935-7) [1972]. La catégorie des cas, Copenhague, Universitetsforlager I Aarhus [repris dans La catégorie des cas, München, Wilhelm Fink Verlag, 1972].

Jackendoff, Ray (1975). « On belief-contexts », Linguistic Inquiry 6(1), 53-93.

Johnson, M. (1987). The body in the mind: the bodily basis of meaning, imagination and reason, Chicago, The University of Chicago Press.

Johnson, Mark (2008). The meaning of the body. Aesthetics of human understanding, Chicago, University of Chicago Press.

Katz, Jerrold J. \& Postal, Paul M. (1964). An integrated theory of linguistic descriptions, Cambridge (Mass.), MIT Press.

Kay, Paul \& McDaniel, Chad (1978). « The linguistic significance of the meanings of basic color terms », Language 54(3), 610-46.

Kleiber, Georges (1990). La sémantique du prototype, Paris, Presses Universitaires de France.

Kleiber, Georges \& Riegel, Martin (1978). « Les grammaires floues », Martin, Robert (dir.), La notion de recevabilité en linguistique, Paris, Klincksieck, 67-123.

Labov, William (1973). « The boundaries of words and their meanings », Bailey, CharlesJames N. \& Shuy, Roger W. (eds), New ways of analysing variation in English, Georgetown University Press, 340-373.

Lakoff, George (1970) [1965]. Irregularity in syntax, New York, Holt Rinehart \& Winston [ $1^{\text {ère }}$ publication en 1965 , sous le titre On the nature of syntactic irregularity, National Science Foundation Report, dissertation, Indiana University].

Lakoff, George, (1970). «Linguistics and natural logic », Synthese 22, 151-271.

Lakoff, George, (1971). «On generative semantics », Steinberg, Danny D. \& Jakobovits, Leon A. (ed.), Semantics: An interdisciplinary reader in philosophy, linguistics and psychology, Cambridge, Cambridge University Press, 232-296.

Lakoff, George (1972a). «Structural Complexity in Fairy Tales », The Study of Man vol. 1., 128-150.

Lakoff, George (1972b). «In Response to: A special supplement. Chomsky's revolution in linguistics », The New York Review of Books June 29.

Lakoff, George (1973a). « Hedges: A study in meaning criteria and the logic of fuzzy concepts », Journal of Philosophical Logic 2, 458-508.

Lakoff, George (1973b). « Fuzzy grammar and the performance / competence terminology game », Papers from the regional meeting of the Chicago linguistic society 9, 271-291.

Lakoff, George (1974). «Syntactic amalgams », Papers from the Tenth Annual Meeting of the Chicago Linguistic Society, 321-344.

Lakoff, George (1976) [1963]. « Toward generative semantics », McCawley, James, Notes from the linguistic underground. Syntax and semantics. Vol. 7, New York, Academic Press, 43-63.

Lakoff, George, (1977). " Linguistic Gestalts », Proceedings of the $13^{\text {th }}$ Meeting of the Chicago Linguistic Society, vol. 13, 236-287. 
Lakoff, George (1982). « Categories: An essay in cognitive linguistics », Linguistic Society of Korea (ed.), Linguistics in the Morning Calm, Seoul, Hanshin, 139-193.

Lakoff, George, (1987). Women, Fire and Dangerous Things: What Categories Reveal about the Mind, Chicago, University of Chicago Press.

Lakoff, George \& Johnson, Mark, (1980). Metaphors We Live By, University of Chicago Press [tr. fr. (1985). Les Métaphores dans la Vie Quotidienne, Paris, Minuit].

Lakoff, George \& Kövecses, Zoltán (1987). « The Cognitive Model of Anger Inherent in American English », Holland, Dorothy \& Quinn, Naomi (eds), Cultural Models in Language and Thought, Cambridge, Cambridge University Press, 195-221.

Lakoff, George \& The Rockridge Institute (2006). Thinking points, New York, Farrar, Strauss and Giroux.

Lakoff, George \& Thompson, Henry (1975). « Introducing cognitive grammar », Proceedings of the First Annual Meeting of the Berkeley Linguistics Society, 295-313.

Lamb, Sydney M. (1964). « The sememic approach to structural semantics », American Anthropologist 66(3/2), 57-78.

Lamb, Sydney M. (1966). Outline of stratificational grammar, Washington, Georgetown University Press.

Lamb, Sydney M. (1971). « The Crooked Path of Progress in Cognitive Linguistics », Georgetown University Monograph Series on Languages and Linguistics 24, 99-123 [Repris dans Makkai, Adam \& Lockwood, David G. (1973). Readings in Stratificational Linguistics, 12-33].

Langacker, Ronald W. (1965). «French interrogatives: A transformational description », Language 41, 587-600.

Langacker, Ronald W. (1968). « Observations on French Possessives », Language 44(1), 51-75.

Langacker, Ronald W. (1969). « On pronominalization and the chain of command », Reibel, David A. \& Schane, Sandford A. (eds), Modern Studies in English, Englewood Cliffs, Prentice Hall, 160-186.

Langacker, Ronald W. (1973) [1967]. Language and its structure. Some fundamental linguistic concepts, New York, Harcourt Brace Jovanovich.

Langacker, Ronald W. (1974a). « The Question of Q ", Foundations of Language 11, 1-37.

Langacker, Ronald W. (1974b). «Movement rules in functional perspective », Language 50, 630-64.

Langacker, Ronald W. (1975). «Functional stratigraphy », Papers from the Parasession on Functionalism, Chicago, Chicago Linguistic Society, 351-397.

Langacker, Ronald W. (1976). « Semantic representations and the linguistic relativity hypothesis », Foundations of Language 14, 307-357.

Langacker, Ronald W. (1978). «The form and meaning of the English auxiliary », Language 54, 853-882.

Langacker, Ronald W., (1979). « Grammar as image », Linguistic Notes from La Jolla, La Jolla, Cal., 6, 87-126.

Langacker, Ronald W. (1981). « The integration of grammar and grammatical change », Indian Linguistics 42, 82-135.

Langacker, Ronald W., (1982). « Space Grammar, analysability, and the English passive », Language 58(1), 22-80.

Langacker, Ronald W. (1986). « An introduction to cognitive grammar », Cognitive Science 10, 1-40.

Langacker, Ronald W. (1987). Foundations of Cognitive Grammar, vol. 1 : Theoretical Prerequisites, Stanford, Stanford University Press. 
Langacker, Ronald W. (1988). « A usage-based model», Rudzka-Ostyn, Brygida (ed.), Topics in cognitive Linguistics, Amsterdam \& Philadelphia, John Benjamins, 127-161.

Langacker, Ronald W. \& Munro, Pamela (1975). « Passives and their meaning », Language 51, 789-830.

Lawler, John (1983). « Review of Metaphors we live by », Language 59(1), 201-207.

Lazard, Gilbert, (2007). «La linguistique cognitive n'existe pas », Bulletin de la Société de Linguistique de Paris 102(1), 3-16.

Legallois, Dominique \& François, Jacques (2011) « La linguistique fondée sur l'usage : Parcours critique », Travaux de Linguistique 62, 7-33.

Levinson, Stephen C. (1992). « Primer for the field investigation of spatial description and conception », Pragmatics 2(1), 5-47.

Lyons, John (1977). Semantics, 2 vol., Cambridge, Cambridge University Press.

Mauthner, Fritz (1923). Beiträge zu einer Kritik der Sprache. Zur Sprachwissenschaft, II $^{\mathrm{er}}$ Bd, Leipzig, F. Meiner.

McCawley, James D. (1976) [1967]. « Meaning and the description of languages », Kotoba no uchú 2, 10-18, 38-48, 51-57 [repris dans McCawley, Grammar and Meaning, New York, Academic Press, 99-120].

McCawley, James D. (1976) [1968a]. « The role of semantics in a grammar », Bach, Emmon \& Harms, Robert T. (ed.), Universals in linguistic theory, New York, Holt Rinehart and Winston, 124-169 [repris dans McCawley (1976). Grammar and Meaning, New York, Academic Press, 59-98].

McCawley, James D. 1976 [1968b] « Lexical insertion in a transformational grammar without deep structure », Papers from the Fourth Regional Meeting of the Chicago Linguistic Society, 71-80 [repris dans McCawley (1976). Grammar and Meaning, New York, Academic Press, 155-166].

McCawley,James D., 1976 [1970] «Where do noun phrases come from? », Jacobs, Roderick A. \& Rosenbaum, Peter S. (eds), Readings in English transformational grammar, Waltham (Mass.), 166-183. [repris dans McCawley (1976). Grammar and Meaning, New York, Academic Press, 133-154].

McCawley, James D. (1976) [1971]. « Prelexical syntax », Georgetown University, Monograph Series on Languages and Linguistics 24, 19-33 [repris dans McCawley (1976). Grammar and meaning, New York, Academic Press, 343-56].

McCawley, James D. (1976a) Grammar and meaning. Papers on syntactic and semantic topics, New York, Academic Press.

McCawley, James D. (1976b) Notes from the linguistic underground. Syntax and semantics. Vol. 7, New York, Academic Press.

Miller, George A. \& Philip N. Johnson-Laird (1976). Language and perception, Cambridge, MA, Harvard University Press.

Minsky, Marvin (1975) «A framework for representing knowledge », Winston, Patrick H. (ed.), The psychology of computer vision, New York, Mc Graw Hill, 211-277.

Nerlich, Brigitte \& Clarke, David (2001). « Mind, meaning and metaphor: the philosophy and psychology of metaphor in $19^{\text {th }}$ century Germany », History of the Human Sciences 14(2), 39-61.

Newmeyer, Frederick, (2 ${ }^{\text {nd }}$ ed.) (1986). Linguistic Theory in America, New York, Academic Press.

Nietzsche, Friedrich (1969) [1873]. « Erkenntnistheoretische Einleitung über Wahrheit und Lüge im außermoralischen Sinne/Introduction théorétique sur la vérité et le mensonge au sens extra-moral », Das Philosophenbuch/Le livre du philosophe (ed. A. KremerMarietti), Paris, Aubier-Flammarion, 170-215. 
Norman, Donald A. \& Rumelhart, David E. (1975). Explorations in cognition, San Francisco, Freeman and co.

Nunberg, Geoffrey D. (1978). The pragmatics of reference, Bloomington, The Indiana University Linguistics Club.

Nyckees, Vincent (2007). « La cognition humaine saisie par le langage : de la sémantique cognitive au médiationnisme », CORELA, numéro thématique Cognition, discours, contextes. [En ligne] http://corela.edel.univ-poitiers.fr/index.php?id=1538.

Ortony, Andrew (ed.) (1979). Metaphor and thought, Cambridge, Cambridge University Press, 19-43.

Ortony, Andrew, Reynolds, Ralph E. \& Arter, Judith A. (1978). « Metaphor: Theoretical and empirical research », Psychological Bulletin 85(5), 919-943.

Parret, Herman (1989). Cognition, the localist hypothesis and back to Kant, Heyvaert, F.J. \& F. Steurs (eds), Worlds Behind Words. Essays in Honour of Professor F.G. Droste on the Occasion of his Sixtieth Birthday, Leuven, Leuven University Press, 37-49.

Partee, Barbara H. (1975). « Montague grammar and transformational grammar », Linguistic Inquiry 6(2), 203-300.

Partee, Barbara H. (1979). « Semantics - mathematics or psychology? » Bäuerle, Rainer, Egli, Urs \& von Stechow, Arnim (eds), Semantics from Different Points of View, Berlin, Springer-Verlag, 1-14.

Partee, Barbara H. (2011). «Formal semantics: Origins, issues, early impact », The Baltic International Yearbook of Cognition, Logic and Communication 6 : Formal semantics and pragmatics. Discourse, context and models, 1-52.

Peeters, Bert (2001). « Does Cognitive Linguistics live up to its name? », Dirven, René, Hawkins, Bruce \& Sandikcioglu, Esra (eds), Language and ideology. Vol. 1 : Theoretical cognitive approaches, Amsterdam, Benjamins, 83-106.

Reddy, Michael J. (1969). « A semantic approach to metaphor », Chicago Linguistic Society Collected Papers, Chicago, Chicago, University Press, 240-251.

Reddy, Michael J. (1979). « The conduit metaphor: A case of frame conflict in our language about language », Ortony, Andrew (ed.), Metaphor and thought, Cambridge, Cambridge University Press, 284-324.

Richards, Ivor A. (1936). The Philosophy of Rhetoric, Oxford, Oxford University Press.

Ricœur, Paul (1975). La métaphore vive, Paris, Le Seuil [tr. angl. 1978, The rule of metaphor: Multi-Disciplinary Studies in the Creation of Meaning in Language, trad. par Robert Czerny, Kathleen McLaughlin \& John Costello, S. J., Toronto, University of Toronto Press].

Rosch, Eleanor Heider (1971). « On the internal structure of perceptual and semantic categories », unpublished paper, Psychology Dept., University of California, Berkeley.

Rosch, Eleanor Heider (1972a). "Universals in color naming and memory », Journal of Experimental Psychology 93, 10-20.

Rosch, Eleanor Heider (1972b). « Probabilities, sampling, and ethnographic method: The case of Dani colour names », Man, New Series, 7(3), 448-466.

Rosch, Eleanor Heider (1973a). « Natural categories », Cognitive Psychology 4, 328-50.

Rosch, Eleanor Heider (1973b). « On the internal structure of perceptual and semantic categories », Moore, Timothy E. (ed.), Cognitive development and the acquisition of language, New York, Academic Press, 111-144.

Rosch, Eleanor (1975). «Universals and cultural specifics in human categorisation », Brislin, Richard W., Bochner, Salomon \& Lonner, Walter J. (eds), Cross-cultural Perspectives of Learning, New York, Sage Publications, 177-206. 
Rosch, Eleanor (1976). « Classification d'objets du monde réel : origines et représentations dans la cognition », Bulletin de Psychologie, numéro spécial La mémoire sémantique, 242-250.

Rosch Eleanor (1977). « Human categorization », Warren, Neil (ed.), Advances in crosscultural psychology, Londres, Academic Press, 1-71.

Rosch Eleanor (1978). « Principles of categorization », Rosch, Eleanor \& Lloyd, Barbara B. (ed.), Cognition and categorization, Hillsdale, NJ, Lawrence Erlbaum Associates, 27-48.

Ruiz de Mendoza Ibáñez, Francisco José (1997). « An interview with George Lakoff », Cuadernos de Filología Inglesa 6/2, 33-52.

Ruppenhofer, Josef, Ellsworth, Michael, Petruck, Miriam, Johnson, Christopher R. \& Scheffczyk, Jan (2006). FrameNet II: Extended theory and practice, Berkeley, FrameNet.

Sandra, Dominiek \& Rice, Sally (1995). « Network analyses of prepositional meaning: mirroring whose mind - the linguist's or the language user's? », Cognitive Linguistics 6(1), 89-130.

Schank, Roger C. \& Abelson, Robert P. (1977). Scripts, plans, goals and understanding, Hillsdale (NJ), Lawrence Erlbaum Associates.

Schön, Donald (1963). The displacement of concepts, London, Tavistock.

Schön, Donald (1979). « Generative metaphor: A perspective on problem-setting in social policy », Ortony, Andrew (ed.), Metaphor and Thought, Cambridge, Cambridge University Press, 284-324.

Searle, John (1979). " Metaphor », Ortony, Andrew (ed.), Metaphor and Thought, Cambridge, Cambridge University Press, 92-123.

Seuren, Pieter, (1998). Western Linguistics: An Historical Introduction, Malden, Blackwell Publishing.

Talmy, Leonard (1972). Semantic structures in English and Atsugewi, dissertation, Berkeley, University of California.

Talmy, Leonard (1975). « Semantics and syntax of motion », Syntax and semantics, vol. 4. Edité par John P. Kimball. New York, Academic Press, 181-238.

Talmy, Leonard (1976). « Semantic causative types », Shibatani, Masayoshi (ed.), Syntax and semantics, vol. 6. The grammar of causative constructions, New York, Academic Press, 43-116.

Talmy, Leonard (1983). « How language structures space », Pick, Herbert L. \& Acredolo, Linda P. (eds), Spatial orientation: Theory, research and application, New York, Plenum Press, 225-282 [repris dans Talmy (2000). Toward a Cognitive Semantics, tome 1, 177-254].

Talmy, Leonard (1985). «Lexicalization patterns: Semantic structure in lexical form », Shopen, Timothy (ed.), Language typology and syntactic description, vol. 3. Cambridge, Cambridge University Press, 57-149 [repris et modifié dans Talmy (2000). Toward a Cognitive Semantics, tome 2, 21-146].

Talmy, Leonard (1988a). «The relation of grammar to cognition », Rudzka-Ostyn, Brygida, Topics in Cognitive Linguistics, Amsterdam, John Benjamins, 165-205 [repris et modifié dans Talmy (2000). Toward a Cognitive Semantics, tome 1, 21-96].

Talmy, Leonard (1988b). "Force dynamics in language and cognition », Cognitive Science 12, 49-100 [repris et modifié dans Talmy (2000). Toward a Cognitive Semantics, tome 1, 409-470].

Talmy, Leonard (1996). « The windowing of attention in language », Shibatani, Masayoshi \& Thompson, Sandra, Grammatical constructions: Their form and meaning, Oxford, Oxford University Press, 235-287 [repris et modifié dans Talmy (2000). Toward a 
Cognitive Semantics, tome 1, 277-309].

Talmy, Leonard (2000). Toward a Cognitive Semantics, 2 vol. Cambridge (Mass.), M.I.T. Press.

Van Dijk, Teun A. (1977). Semantic macrostructures and knowledge frames in discourse comprehension, Just, Marcel Adam \& Carpenter, Patricia A. (eds), Cognitive processes in comprehension, Hillsdale (NJ), Lawrence Erlbaum Associates, 3-32.

Vandeloise, Claude (1986). L'espace en français, Paris, Le Seuil.

Vico, Giambattista (1744). Principi di scienza nuova, Naples, Stamperia Muziana.

Whorf, Benjamin Lee (1956) [1939]. « Gestalt technique of stem composition in Shawnee», Voegelin, Charles, Frederick, 1906-1986, Shawnee stems and the Jacob P. Dunn Miami dictionary, Indianapolis, Indiana Historical Society [repris dans Whorf, B. (1956). Language, Thought, and Reality, 160-72].

Whorf, Benjamin Lee (1956). Language, Thought, and Reality, Cambridge (Mass.), The M.I.T. Press.

Zadeh, Lofti (1965). « Fuzzy sets », Information and Control 8, 338-53.

Zager, David (1980). A real-time process model of morphological change, Buffalo, SUNY dissertation. 